\title{
Strong Regular Embeddings of Deligne-Mumford Stacks and Hypertoric Geometry
}

\author{
DAN EDIDIN
}

\begin{abstract}
We introduce the notion of strong regular embeddings of Deligne-Mumford stacks. These morphisms naturally arise in the related contexts of generalized Euler sequences and hypertoric geometry.
\end{abstract}

\section{Introduction}

Let $G$ be a finite group acting on affine schemes $X=\operatorname{Spec} A$ and $Y=\operatorname{Spec} B$, and let $f: Y \rightarrow X$ be a $G$-equivariant morphism. Since $f$ is $G$-equivariant, there is an induced map of invariant subrings $A^{G} \rightarrow B^{G}$ corresponding to a morphism of quotients $g: Y / G \rightarrow X / G$. Certain algebro-geometric properties of the morphism $f$ (typically related to finiteness) are automatically preserved by the morphism $g$. For example, if $f$ is finite, then the induced morphism of quotients $g: Y / G \rightarrow X / G$ is also finite. Likewise, if $|G|$ is a unit in $\operatorname{Spec} B$ and $f: Y \rightarrow X$ is a closed embedding, then $g: Y / G \rightarrow X / G$ is as well.

On the other hand, many properties of the morphism $f$ will not descend. If $f$ is flat or smooth, the induced morphism of quotients need not be. Instead, we can impose additional conditions on the actions of $G$ on $X$ and $Y$ to ensure that a property of morphisms of schemes does descend to the quotient. Two obvious conditions that suffice are that $G$ act freely or that $G$ act trivially on both spaces.

Note, however, that these conditions are not necessary. For example, if $Y=$ $X \times Z$ and $G$ acts trivially on $Z$, then $Y / G=X / G \times Z$, so the flat projection $Y \rightarrow X$ descends to a flat projection $Y / G \rightarrow X / G$, and the diagram

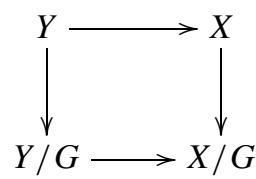

is Cartesian.

This is an example of a stabilizer preserving morphism, meaning that for every point $y \in Y$, the map of stabilizers $\operatorname{Stab}_{y} Y \rightarrow \operatorname{Stab}_{f(y)} X$ is an isomorphism of groups. A folklore theorem [KM, cf. Lemma 6.3] states that an étale stabilizer-

Received April 15, 2015. Revision received December 21, 2015.

The author's research was supported in part by Simons collaboration grant 315460 . 
preserving morphism is strongly étale. This means that the morphism of quotients $Y / G \rightarrow X / G$ and the diagram

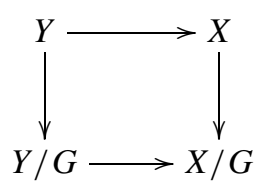

is Cartesian.

In this paper we turn our attention to the problem of determining when a regular embedding $i: Y \hookrightarrow X$ descends to a regular embedding of quotients $j: Y / G \hookrightarrow X / G$ (necessarily of codimension $d$ since $\operatorname{dim} Y=\operatorname{dim} Y / G$ and $\operatorname{dim} X=\operatorname{dim} X / G)$. The $G$-equivariant morphism $Y \rightarrow X$ is a local model for a morphism $\iota: \mathcal{Y} \rightarrow \mathcal{X}$ of Deligne-Mumford stacks. We say that a morphism of Deligne-Mumford stacks is a strong regular embedding if the induced morphism of coarse spaces $Y \rightarrow X$ is a regular embedding and the diagram of stacks and spaces

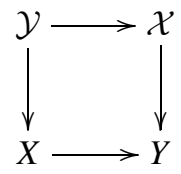

is Cartesian.

Although any immersion of stacks is stabilizer preserving, not every regular embedding is strong (Example 3.1). Our first result, Theorem 2.2, is a characterization of strong regular embeddings. When $\mathcal{Y} \hookrightarrow \mathcal{X}$ is a strong regular embedding of smooth stacks, we use Artin's approximation theorem [Art] to prove that the induced morphism of coarse spaces $Y \hookrightarrow X$ is étale locally the section of a smooth morphism. As a corollary, we prove that if $\tilde{X} \rightarrow X$ is a functorial resolution of singularities (in the sense of [Kol]), then the fiber product $\tilde{Y}=Y \times_{X} \tilde{X}$ is a resolution of singularities of $Y$.

We also prove that the pullback on Chow groups $(I \iota)^{*}: \mathrm{CH}^{*}(I \mathcal{X}) \rightarrow$ $\mathrm{CH}^{*}(I \mathcal{Y})$ commutes with the Chen-Ruan orbifold product, thereby giving a ring homomorphism of orbifold Chow rings $\mathrm{CH}_{\text {orb }}^{*}(\mathcal{X}) \rightarrow \mathrm{CH}_{\text {orb }}^{*}(\mathcal{Y})$.

\subsection{Applications}

Strong regular embeddings arise in two related contexts, generalized Euler sequences and hypertoric geometry.

1.1.1. Generalized Euler Sequences. Let $T$ be a torus acting with finite unramified inertia on a smooth scheme $X$. The quotient stack $\mathcal{X}=[X / T]$ is a smooth Deligne-Mumford stack whose coarse space is a scheme by Sumihiro's theorem [Sum]. The cotangent bundle $T^{*} \mathcal{X}$ fits into a generalized Euler sequence of vector bundles on $\mathcal{X}$

$$
0 \rightarrow T^{*} \mathcal{X} \rightarrow\left[T^{*} X / T\right] \rightarrow \mathcal{X} \times \operatorname{Lie}(T)^{*} \rightarrow 0
$$


which implies by Theorem 2.2 that the inclusion $T^{*} \mathcal{X} \rightarrow\left[T^{*} X / T\right]$ is a strong regular embedding. We prove that if $\mathbf{M}$ and $\mathbf{N}$ are the coarse spaces of $T^{*} \mathcal{X}$ and $\left[T^{*} X / T\right]$, respectively, then the regular embedding $i: \mathbf{M} \rightarrow \mathbf{N}$ induces an isomorphism of integral Chow groups $i^{*}: \mathrm{CH}^{*}(\mathbf{N}) \rightarrow \mathrm{CH}^{*}(\mathbf{M})$. We also conjecture that if $\tilde{\mathbf{N}} \rightarrow \mathbf{N}$ is a functorial resolution of singularities, then the pullback along the morphism $\tilde{\mathbf{M}} \rightarrow \tilde{\mathbf{N}}$ obtained by base change induces an isomorphism of integral Chow rings $\mathrm{CH}^{*}(\tilde{\mathbf{N}}) \rightarrow \mathrm{CH}^{*}(\tilde{\mathbf{N}})$. Finally, we prove that the pullback $(I \iota)^{*}: \mathrm{CH}^{*}\left(I\left[T^{*} X / T\right]\right) \rightarrow \mathrm{CH}^{*}\left(I T^{*} \mathcal{X}\right)$ induces an isomorphism of orbifold Chow rings.

1.1.2. Hypertoric Stacks. Hypertoric varieties were first defined by Bielawski and Dancer [BD] and give a large and interesting class of algebraic symplectic manifolds with complete hyper-Kähler metrics. Algebraic symplectic manifolds naturally occur in a variety of mathematical contexts including representation theory (Springer resolutions, Nakajima quiver varieties, Slodowy slices), string theory (moduli spaces of Higgs bundles), and mirror symmetry (Gromov-Witten theory of $\mathrm{K} 3$ surfaces and $T^{*} \mathbb{P}^{1}$ ).

The theory was further developed by a number of authors including Hausel and Sturmfels [HS] and Proudfoot [Pro2; Pro1].

Let $T$ be a torus of rank $d$, and let $V$ be an $n$-dimensional representation of $T$ with $d \times n$ weight matrix $A=\left(a_{i j}\right)$. There is a natural algebraic moment map $\mu: V \times V^{*} \rightarrow \operatorname{Lie}(T)^{*}$, and the hypertoric variety $\mathbf{Y}(A, \theta)$ is defined as the double reduction $\left(V \times V^{*}\right) / / / / T$, where the first reduction is an algebraic symplectic reduction with respect to $\mu$, and the second reduction is a GIT quotient with respect to the torus action linearized with respect to a character $\theta \in X(T)$.

The hypertoric variety $\mathbf{Y}(A, \theta)$ naturally embeds in a Lawrence toric variety $\mathbf{X}\left(A^{ \pm}, \theta\right)$, which is the GIT quotient $\left(V \times V^{*}\right) / / T$. Following Jiang and Tseng [JT1], we refer to the corresponding quotient stack $\mathcal{Y}(A, \theta)\left(\operatorname{resp} . \mathcal{X}\left(A^{ \pm}, \theta\right)\right)$ as a hypertoric stack (resp. Lawrence toric stack). If the character $\theta$ is generic, then the stacks $\mathcal{Y}(A, \theta)$ and $\mathcal{X}\left(A^{ \pm}, \theta\right)$ are Deligne-Mumford, and the corresponding varieties are their coarse spaces.

In Section 5 we prove that the embedding $\mathcal{Y}(A, \theta) \hookrightarrow \mathcal{X}\left(A^{ \pm}, \theta\right)$ is a strong regular embedding. This implies that the morphism $\mathbf{Y}(A, \theta) \rightarrow \mathbf{X}\left(A^{ \pm}, \theta\right)$ is a regular embedding which is étale locally a section of smooth morphism, so that any functorial resolution of singularities of the Lawrence toric stack $\mathbf{X}\left(A^{ \pm}, \theta\right)$ pulls back to resolution of singularities of the hypertoric variety $\mathbf{Y}(A, \theta)$.

We also prove (Theorem 5.5) that the inclusion morphism $\iota: \mathcal{Y}(A, \theta) \hookrightarrow$ $\mathcal{X}\left(A^{ \pm}, \theta\right)$ induces an isomorphism of integral Chow rings $\mathrm{CH}^{*}\left(\mathcal{X}\left(A^{ \pm}, \theta\right)\right) \rightarrow$ $\mathrm{CH}^{*}(\mathcal{X}(A, \theta))$ and integral Chow groups $\mathrm{CH}^{*}\left(\mathbf{X}\left(A^{ \pm}, \theta\right)\right) \rightarrow \mathrm{CH}^{*}(\mathbf{Y}(A, \theta))$.

As a corollary of Theorems 5.5 and 2.7, we also prove (Corollary 2.9) that the inclusion of inertia stacks $I \mathcal{Y}(A, \theta) \rightarrow I \mathcal{X}\left(A^{ \pm}, \theta\right)$ induces an isomorphism of integral orbifold Chow rings. With rational coefficients, this result was previously obtained by Jiang and Tseng [JT1]. 


\subsection{Conventions and Notation}

1.2.1. Generalities on Stacks. Unless otherwise stated, we work with DeligneMumford stacks of finite type (and hence finite presentation) over a Noetherian scheme $S$.

Let $\mathcal{X}$ be a Deligne-Mumford stack. If $T$ is an $S$-scheme and $T \stackrel{x}{\rightarrow} \mathcal{X}$ is a $T$ valued point, then we use the notation $\operatorname{Aut}(x)$ to denote the $T$-group $\operatorname{Isom}_{T}(x, x)$.

If $\mathcal{X}$ is a Deligne-Mumford stack, then we denote by $I \mathcal{X}$ the inertia stack $\mathcal{X} \times \mathcal{X} \times \mathcal{X} \mathcal{X}$ where the fiber product is taken with respect to the diagonal $\mathcal{X} \rightarrow$ $\mathcal{X} \times \mathcal{X}$. Because $\mathcal{X}$ is Deligne-Mumford, the natural morphism $I \mathcal{X} \rightarrow \mathcal{X}$ is unramified. The fiber of $I \mathcal{X} \rightarrow \mathcal{X}$ over a $T$-valued point $T \stackrel{x}{\rightarrow} \mathcal{X}$ is the $T$-group $\operatorname{Aut}(x)$. We denote by $I^{2} \mathcal{X}$ the double inertia stack defined as the fiber product $I \mathcal{X} \times \mathcal{X} I \mathcal{X}$. Note that $I^{2} \mathcal{X}$ is not in general equivalent to the stack $I(I \mathcal{X})$.

A Deligne-Mumford stack $\mathcal{X}$ has finite inertia if the morphism $I \mathcal{X} \rightarrow \mathcal{X}$ is finite. The stack is separated if the diagonal $\mathcal{X} \rightarrow \mathcal{X} \times \mathcal{X}$ is finite. Any separated stack has finite inertia, but the converse need not hold.

Following [AOV], we say that a Deligne-Mumford stack is tame if it has finite inertia and if for every geometric point $\operatorname{Spec} k \stackrel{x}{\rightarrow} \mathcal{X}$ of $\mathcal{X}$, the finite group $\operatorname{Aut}(x)$ is linearly reductive over Spec $k$. This is equivalent to saying that $|\operatorname{Aut}(x)|$ is prime to the characteristic of $k$.

An algebraic space $X$ is a coarse space of a stack $\mathcal{X}$ if there is a morphism $\mathcal{X} \stackrel{\pi}{\rightarrow} X$ such that

(i) $\pi$ is initial for maps from $\mathcal{X}$ to algebraic spaces, that is, for any morphism $f: \mathcal{X} \rightarrow Z$ with $Z$ an algebraic space, there is a unique morphism of algebraic spaces $g: X \rightarrow Z$ such that the morphism $f$ factors as $g \circ \pi$,

(ii) $\pi$ induces a bijection between geometric points of $\mathcal{X}$ and geometric points of $X$.

The universal property (i) implies that the coarse space is unique up to (unique) isomorphism of algebraic spaces.

A fundamental theorem of Keel and Mori [KM] implies that any stack with finite inertia has a coarse space $X$.

A stack $\mathcal{X}$ is a quotient stack if it is equivalent to a stack of the form $[X / G]$ where $X$ is an algebraic space and $G$ is flat closed subgroup scheme of $\operatorname{GL}_{n}(S)$ for some $n$. Equivalently, we may assume that $\mathcal{X}=\left[X^{\prime} / \operatorname{GL}_{n}(S)\right]$ where $X^{\prime}$ is the algebraic space $\left(X \times \mathrm{GL}_{n}(S)\right) / G$.

If $\mathcal{X}=[X / G]$ is a quotient stack, then the coarse space of $\mathbf{X}$ of $\mathcal{X}$ is the geometric quotient $X / G$ in the category of algebraic spaces.

1.2.2. Chow Groups. If $X$ is a scheme defined over a field, then we denote by $\mathrm{CH}_{d}(X)$ the group of $d$-dimensional cycles modulo rational equivalence. Likewise, we denote by $\mathrm{CH}^{d}(X)$ the group of codimension- $d$ cycles modulo rational equivalence. When $X$ is equidimensional of dimension $n, \mathrm{CH}_{d}(X)=\mathrm{CH}^{n-d}(X)$. Set $\mathrm{CH}_{*}(X)=\bigoplus_{d} \mathrm{CH}_{d}(X)$ and $\mathrm{CH}^{*}(X)=\bigoplus_{d} \mathrm{CH}^{d}(X)$. If $f: Y \rightarrow X$ is proper, then there is a pushforward $f_{*}: \mathrm{CH}_{d}(Y) \rightarrow \mathrm{CH}_{d}(X)$. Likewise if $f$ is flat, or an lci morphism, then there is a pullback $f^{*}: \mathrm{CH}^{d}(X) \rightarrow \mathrm{CH}^{d}(Y)$. When $X$ is 
smooth, the diagonal is a regular embedding, and pullback along the diagonal defines a graded ring structure on $\mathrm{CH}^{*}(X)$.

This definition of Chow groups formally extends to algebraic spaces.

If $G$ is a linear algebraic group acting on an algebraic space $X$, then we can define equivariant Chow groups [EG1]. The group $\mathrm{CH}_{G}^{d}(X)$ is defined as $\mathrm{CH}^{d}((X \times U) / G)$ where $U$ is an open set in a representation $V$ of $G$ such that $G$ acts freely on $U$ and $\operatorname{codim}(V \backslash U)>d$. This definition is independent of the representation $V$ and the open set $U$.

If $\mathcal{X}$ is a Deligne-Mumford stack, then $\mathrm{CH}^{d}(\mathcal{X})$ denotes the Chow group in codimension $d$ defined by Kresch in [Kre]. Typically, we will work with quotient stacks $\mathcal{X}=[X / G]$. In this case, $\mathrm{CH}^{d}(\mathcal{X})=\mathrm{CH}_{G}^{d}(X)$ where $\mathrm{CH}_{G}^{d}(X)$ denotes the equivariant Chow group defined before. Note that $\mathrm{CH}^{d}(\mathcal{X})$ can be nonzero for $d>\operatorname{dim} \mathcal{X}$, although this group will be torsion. Likewise, the group $\mathrm{CH}_{d}(\mathcal{X})$ can be nonzero for negative $d$.

Bloch defined for a scheme $X$, higher Chow groups $\mathrm{CH}_{d}(X, k)$ parameterizing cycles of dimension $d+k$ on $X \times \Delta^{k}$, which intersect the faces of $\Delta^{k}$ transversely. (Here $\Delta^{k}$ denotes the algebraic $k$-simplex Spec $k\left[t_{0}, \ldots, t_{k}\right] /\left(t_{0}+\cdots+t_{k}-1\right)$.) In this definition $\mathrm{CH}^{d}(X, 0)=\mathrm{CH}^{d}(X)$.

A key property of higher Chow groups proved by Bloch [Blo] and Levine [Lev] is that if $X$ is an arbitrary scheme and $Z \subset X$ is a closed subscheme with complement $U$, then there is a localization long exact sequence

$$
\cdots \rightarrow \mathrm{CH}_{d}(Z, k) \rightarrow \mathrm{CH}_{d}(X, k) \rightarrow \mathrm{CH}_{d}(U, k) \rightarrow \cdots
$$

extending the classical localization sequence

$$
\mathrm{CH}_{d}(Z) \rightarrow \mathrm{CH}_{d}(X) \rightarrow \mathrm{CH}_{d}(U) \rightarrow 0
$$

for ordinary Chow groups.

1.2.3. Regular Embeddings. A closed embedding of schemes $Y \hookrightarrow X$ is a regular embedding if $Y$ is Zariski locally cut out by a regular sequence of functions on $X$. This notion readily generalizes to Deligne-Mumford stacks by replacing Zariski locally with étale locally. Precisely, a closed embedding of Deligne-Mumford stacks $\mathcal{Y} \hookrightarrow \mathcal{X}$ is a regular embedding if there is an étale surjective morphism $U \rightarrow \mathcal{X}$ with $U$ a scheme such that morphism of schemes obtained by base change along $U \rightarrow \mathcal{X}$ is a regular embedding.

\section{Definitions and Statements of Results}

Definition 2.1. A morphism of Deligne-Mumford stacks $\iota: \mathcal{Y} \rightarrow \mathcal{X}$ is said to be a strong regular embedding if the induced morphism of coarse spaces $i: Y \rightarrow X$ is a regular embedding and the diagram

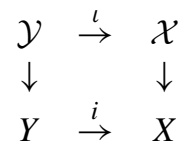

is Cartesian. 
Our first result is the following characterization of strong regular embeddings:

Theorem 2.2. Let $\iota: \mathcal{Y} \rightarrow \mathcal{X}$ be a regular embedding of tame Deligne-Mumford stacks, and let $Y \rightarrow X$ be the corresponding embedding of coarse spaces. The following conditions are equivalent:

(i) $\iota: \mathcal{Y} \rightarrow \mathcal{X}$ is a strong regular embedding.

(ii) There is a stratification of $\mathcal{Y}$ by locally closed substacks such that the normal bundle $N_{\iota}$ restricted to each stratum is trivial.

(ii') If $Y$ is a scheme, then $N_{\iota}$ is locally trivial in the Zariski topology on $\mathcal{Y}$.

(iii) For every geometric point Spec $k \stackrel{y}{\rightarrow} \mathcal{Y}$, the pullback of $\left(N_{\iota}\right)_{y}$ is a trivial representation of the inertia group $G_{y}=\operatorname{Aut}(y)$.

(iv) For every geometric point $\operatorname{Spec} k \stackrel{y}{\rightarrow} \mathcal{Y}$, there is a strongly étale morphism $\left[U / G_{y}\right] \rightarrow \mathcal{X}$ with $U$ affine such that normal bundle to the regular embed$\operatorname{ding} \mathcal{Y} \times \mathcal{X}[U / G] \hookrightarrow[U / G]$ is trivial.

(v) For every geometric point $\operatorname{Spec} k \stackrel{y}{\rightarrow} \mathcal{Y}$, there is a strongly étale morphism $\left[U / G_{y}\right] \rightarrow \mathcal{X}$ where $U$ is an affine scheme such that $\mathcal{Y} \times \mathcal{X} U$ is defined by a $G$-fixed regular sequence in $\mathcal{O}(U)$.

\subsection{Strong Regular Embeddings and Resolutions of Singularities}

Given a strong regular embedding $\iota: \mathcal{Y} \rightarrow \mathcal{X}$ of smooth Deligne-Mumford stacks, we obtain a regular embedding of the possibly singular coarse spaces $Y \rightarrow X$.

Our next result shows that this morphism is rather distinguished among regular embeddings in that it is étale locally a section of a smooth morphism.

Definition 2.3. A regular embedding $Y \stackrel{i}{\hookrightarrow} X$ with the following local structure is called a tubular regular embedding: For each point of $y \in Y \subset X$, there is a commutative diagram

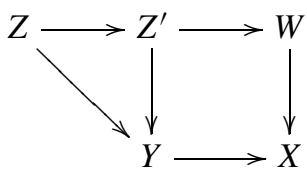

where $W \rightarrow X$ and $Z \rightarrow Y$ are étale neighborhoods of $y, Z \rightarrow Z^{\prime}$ is étale, and $Z^{\prime} \rightarrow W$ is a section of a smooth morphism.

THEOREM 2.4. Let $\mathcal{Y} \rightarrow \mathcal{X}$ be a strong regular embedding of smooth DeligneMumford stacks. Then the induced regular embedding $Y \rightarrow X$ is a tubular regular embedding.

As an application of Theorem 2.4, we can show that the induced morphisms of coarse moduli spaces has a strong functorial property with respect to resolutions of singularities. 
COROLlary 2.5. Let $\iota: \mathcal{Y} \rightarrow \mathcal{X}$ be a strong regular embedding of smooth tame Deligne-Mumford stacks defined over a perfect field $k$. Then, if $\tilde{X}$ is a canonical (functorial) resolution of singularities of $X$, then $Y \times_{X} \tilde{Y}$ is a resolution of singularities of $Y$.

REMARK 2.6. In a recent preprint, Buonerba [Buo] proved functorial resolution of tame quotient singularities in arbitrary characteristic. This implies that the coarse space of every tame, smooth Deligne-Mumford stack admits a functorial resolution of singularities.

\subsection{Strong Regular Embeddings and Orbifold Products}

For stacks defined over a field, we also obtain the following result about orbifold products. See Section 3.3 for the notation. The proof will be given in Section 3.3.

TheOREM 2.7. Let $\iota: \mathcal{Y} \rightarrow \mathcal{X}$ be a strong regular embedding of smooth tame Deligne-Mumford stacks, and let Iı:IY $\rightarrow I \mathcal{X}$ be the induced morphism of inertia stacks. The pullback $(I \iota)^{*}: \mathrm{CH}^{*}(I \mathcal{X}) \rightarrow \mathrm{CH}^{*}(I \mathcal{Y})$ commutes with the orbifold products on $\mathrm{CH}^{*}(I \mathcal{X})$ and $\mathrm{CH}^{*}(I \mathcal{Y})$.

As an application, we obtain the following corollaries, which will be proved in Section 4 and Section 5, respectively.

Corollary 2.8. Let $T$ be a torus acting on a smooth variety $X$ so that $\mathcal{X}=$ $[X / T]$ is a tame Deligne-Mumford stack. Then, the pullback $(I \iota)^{*}: \mathrm{CH}^{*}\left(I\left[T^{*} \times\right.\right.$ $X / T]) \rightarrow \mathrm{CH}^{*}\left(I T^{*} \mathcal{X}\right)$ induces an isomorphism of orbifold Chow rings $\mathrm{CH}_{\text {orb }}^{*}\left(\left[T^{*} X / T\right]\right) \rightarrow \mathrm{CH}_{\text {orb }}^{*}\left(T^{*} \mathcal{X}\right)$.

Corollary 2.9. If $\mathcal{Y}(A, \theta)$ is a hypertoric stack ${ }^{1}$ and $\mathcal{X}(A, \theta)$ is the associated Lawrence toric stack, then the pullback on Chow groups $(I \iota)^{*}: \mathrm{CH}^{*}(\mathcal{X}(A, \theta)) \rightarrow$ $\mathrm{CH}^{*}(\mathcal{Y}(A, \theta))$ induces an isomorphism of orbifold Chow rings.

The analogous results also hold for orbifold $K$-theory.

REMARK 2.10. With rational coefficients, Corollary 2.9 was proved by direct calculation by Jiang and Tseng in [JT1, Thm. 3.10].

\section{Proofs of Theorems}

\subsection{Proof of Theorem 2.2}

(i) $\Longrightarrow$ (ii), (ii ${ }^{\prime}$. Since the relative dimension of the morphism $\mathcal{Y} \stackrel{\iota}{\rightarrow} \mathcal{X}$ is necessarily the same as the relative dimension of the morphism $Y \stackrel{i}{\rightarrow} X$, the normal bundle $N_{\iota}$ is the pullback of the normal bundle $N_{i}$. If $Y$ is an algebraic space, then it has a stratification by schemes, and on each open stratum, $N_{i}$ is locally trivial. Refining the stratification if necessary, we obtain one where $N_{i}$ is trivial on each open stratum. Pulling back to $\mathcal{Y}$ gives the desired conclusion about $N_{l}$. (Note that

\footnotetext{
${ }^{1}$ See Section 5 for notation and definitions.
} 
we are using the fact that tame quotients are preserved by base change along the strata.) In the case where $Y$ is a scheme, then $N_{i}$ is locally trivial in the Zariski topology, and (ii') follows.

(ii) $\Longrightarrow$ (iii). Let $\operatorname{Spec} k \stackrel{y}{\rightarrow} \mathcal{Y}$ be a geometric point of $\mathcal{Y}$. Our hypothesis (ii) implies that the pullback of $N_{\iota}$ to $B G_{y}$ is trivial. This means that the fiber of $N_{\iota}$ at $y$ is a trivial $G_{y}$-module, which is assertion (iii).

(iii) $\Longrightarrow$ (iv). By the local structure theorem for Deligne-Mumford stacks (cf. [AOV, Lemma 2.2.3]), every geometric point $y \rightarrow \mathcal{Y}$ has a strongly étale neighborhood isomorphic to $\left[U / G_{y}\right]$ where $U$ is an affine scheme and $G_{y}=\operatorname{Aut}_{y}(\mathcal{Y})$. The pullback $N$ of $N_{\iota}$ to $\left[U / G_{y}\right]$ corresponds to a $G_{y}$-equivariant vector bundle $N$ on the affine scheme $U$. Let $O$ be a point of $U$ mapping to $y$ so that $O$ is fixed by $G_{y}$. In a neighborhood of the fixed point $O$, the bundle $N$ is nonequivariantly trivial. Taking the intersection over all $g \in G_{y}$ of the translates of this neighborhood, we obtain a $G_{y}$-invariant neighborhood of $O$ such that the restriction of $N$ to this neighborhood is nonequivariantly trivial. Replacing $U$ with this neighborhood, we may assume that $N$ decomposes as $\bigoplus_{V} V \otimes \mathcal{O}_{U}^{r_{V}}$, where the sum is over the irreducible representations of $G_{y}$. By hypothesis the fiber of $N$ at the fixed point $O$ is a trivial representation. So $N$ must be globally trivial as a $G_{y}$-equivariant bundle.

(iv) $\Longrightarrow$ (v). Again, let $O$ be a fixed point for the $G_{y}$ action that maps to $y$, and let $V=\mathcal{Y} \times \mathcal{X} U$. Then $\mathcal{Y} \times \mathcal{X}\left[U / G_{y}\right]=\left[V / G_{y}\right]$. Since $O$ is a fixed point, the local ring $A=\mathcal{O}_{U, O}$ has an induced action of $G_{y}$. Let $B$ denote the local ring $\mathcal{O}_{V, G}$, so $B=A / I$. We wish to show that $I$ is generated by a $G_{y}$-fixed regular sequence. By assumption $G_{y}$ acts trivially on the $B$-module $I / I^{2}$. Then $I / I^{2}$ is free with basis $\bar{x}_{1}, \ldots, \bar{x}_{r}$, each of which is $G_{y}$-fixed. Let $x_{1}, \ldots, x_{m}$ be lifts of these elements to a regular sequence in $A$. By construction $g x_{i} \equiv$ $x_{i} \bmod I^{2}$. Since $G$ is linearly reductive, the $G$-module has a Reynolds operator $\rho$. Let $y_{i}=\rho\left(x_{i}\right)$; then $y_{i} \equiv x_{i} \bmod I^{2}$, so the $y_{i}$ form a $G_{y}$-fixed regular sequence that generate $I$.

(v) $\Longrightarrow$ (i). Since $[U / G] \rightarrow \mathcal{X}$ is strongly étale, the corresponding morphism $U / G \rightarrow X$ is étale, and to check that $\iota: \mathcal{Y} \rightarrow \mathcal{X}$ is a strong regular embedding, it suffices to check that the morphism $[V / G] \rightarrow[U / G]$ is a strong regular embedding where $V=\mathcal{Y} \times \mathcal{X} U$.

As before, assume that $U=\operatorname{Spec} A$ and $V=\operatorname{Spec} A / I$. Then we must show that the kernel of the morphism of invariant rings $A^{G} \rightarrow(A / I)^{G}$ is generated by a regular sequence and that $A / I=A^{G} \otimes_{A^{G}}(A / I)^{G}$.

Let $x_{1}, \ldots, x_{r} \in A$ be a regular sequence of $G$-fixed elements that generate $I$. Then these elements also generate the invariant ideal $I^{G}$. Since $G$ is linearly reductive, $(A / I)^{G}=A^{G} / I^{G}$ as $A^{G}$-modules. Also, since $A^{G}$ is a subring of $A$, the sequence $x_{1}, \ldots, x_{n}$ is also regular in $A^{G}$. Finally, since $I$ is generated by $G$-fixed elements, $I^{G} A=I$, so $A / I=A \otimes_{A^{G}}(A / I)^{G}$, as claimed. 
EXAMPLE 3.1. We illustrate the failure of the theorem when $\iota$ is not a strong regular embedding. Let $Y=\mathbb{A}^{2}$ with the action of $G= \pm 1$ given by $(-1)(x, y)=$ $(-x,-y)$, and let $X=\{(0,0)\}$. Then the morphism of quotient stacks $B G=$ $[X / G] \rightarrow[Y / G]$ is a regular embedding. However, the induced morphism of coarse moduli spaces is the inclusion of the singular point in the affine quadric cone Spec $k\left[x^{2}, y^{2}, x y\right]$.

In this example, $I$ is not generated by a $G$-fixed regular sequence, and $I^{G}$ is generated by $\left(x^{2}, x y, y^{2}\right)$, which do not form a regular sequence in the invariant subring $k\left[x^{2}, x y, y^{2}\right]$. The normal bundle to $\mathcal{Y}$ in $\mathcal{X}$ is the vector bundle on $B G$ corresponding to the nontrivial two-dimensional representation of $G$ with weights $(-1,-1)$.

On the other hand, if we consider the action given by $(-1)(a, b)=(-a, b)$ and let $Y=Z(y)$, then $Y$ is defined by a $G$-fixed regular sequence, and $I=(y)=$ $I^{G}$, so that $A / I=\operatorname{Spec} k[x]$ is obtained by extension of scalars from $A^{G} / I^{G}=$ Spec $k\left[x^{2}\right]$.

EXAMPLE 3.2. The tameness assumption is crucial, so that the group $G$ is linearly reductive. Let $k$ be field of characteristic 2 , and let $G=\mathbb{Z}_{2}$ act on $k[x, y]$ by exchanging coordinates. Let $I=(x+y)$. Then $I$ is generated by a $G$-fixed regular sequence. However, the sequence of $G$-modules $0 \rightarrow I \rightarrow A \rightarrow A / I$ does not remain exact after taking $G$-invariants since the map $A^{G}=k[x+y, x y] \rightarrow$ $(A / I)^{G}=k[x, y] /(x+y)$ is not surjective. In particular, the map $\operatorname{Spec}(A / I)^{G} \rightarrow$ Spec $A^{G}$ is not a closed embedding. Hence, the regular embedding of stacks $[(\operatorname{Spec} A / I) / G] \rightarrow[(\operatorname{Spec} A) / G]$ is not a strong regular embedding.

\subsection{Proofs of Theorem 2.4 and Corollary 2.5}

Proof of Theorem 2.4. Let $y \rightarrow \mathcal{Y} \hookrightarrow \mathcal{X}$ be a geometric point. By the local structure theorem for tame Deligne-Mumford stacks there is a strongly étale neighborhood $\left[U / G_{y}, O\right] \rightarrow(\mathcal{X}, y)$ where $U=\operatorname{Spec} A$ is affine and $O \in U$ is $G_{y}$-fixed.

Since the morphism $\mathcal{Y} \rightarrow \mathcal{X}$ is representable and affine, the inverse image $V$ of $\mathcal{Y}$ in $U$ is affine, and the morphism $\left(\left[V / G_{y}\right], O\right) \rightarrow(\mathcal{Y}, y)$ is also strongly representable, and we have a Cartesian square

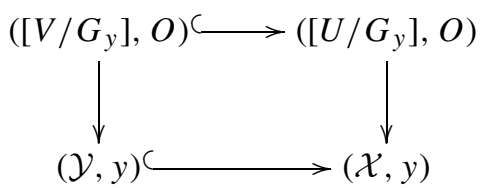

where the vertical maps are strongly étale.

Thus, we are reduced to the case that $\mathcal{X}=[U / G]$ with $U=\operatorname{Spec} A$ affine, $G$ a finite reductive group, and $\mathcal{Y}=[V / G]$ where $V=\operatorname{Spec} A / I$ is a closed subscheme whose ideal $I=\left(f_{1}, \ldots, f_{r}\right)$ is generated by a $G$-invariant regular sequence. Since $O \in U$ in assumed to be $G$-fixed, there is an induced action of $G$ on $\hat{A}$, the completion of $A$ at the maximal ideal of $O$. Since $V$ is smooth over the ground scheme, there is an isomorphism of $G$-algebras $\hat{A} \simeq \widehat{A / I}\left[\left[\bar{f}_{1}, \ldots, \bar{f}_{r}\right]\right]$ 
where the $\bar{f}_{i}$ is any lift of $f_{i}$ to $A$. Since $G$ acts trivially on the $f_{i}$, we obtain an isomorphism $(\hat{A})^{G} \simeq(\widehat{A / I})^{G}\left[\left[\bar{f}_{1}, \ldots, \bar{f}_{r}\right]\right]$.

We claim that $(\hat{A})^{G}=\widehat{A^{G}}$, where the completion of $A^{G}$ is taken at the contraction of the maximal ideal $\mathfrak{m}$ corresponding to $O \in \operatorname{Spec} A$. To see this, we argue as follows. Since $G$ is linearly reductive, there is an isomorphism of $A^{G}$-algebras $(\tilde{A})^{G}=\widehat{A^{G}}$, where $\tilde{A}$ is the completion of $A$ at $\mathfrak{m} \cap A^{G}$ [EG2, Lemma 4.2]. Next, observe that since $O$ is a $G$-fixed point, the inverse image of $\mathfrak{m} \cap A^{G}$ in Spec $A$ is the single point $O$ This means that $\left(\mathfrak{m} \cap A^{G}\right) A$ is $\mathfrak{m}$-primary and the two ideals generate the same topology on $A$. Hence, $\tilde{A}=\hat{A}$.

Thus, the completion of the local ring of $X=U / G$ at the image of $\mathrm{O}$ is isomorphic to a formal power series ring over the completion of the local ring of $Y=V / G$ at the image $P$ of $O$ in $Y$. Applying Artin's étale approximation theorem [Art, Cor. 2.6], there are a scheme $W$ and étale morphisms $W \rightarrow X$ and $W \rightarrow Y \times \mathbb{A}^{r}$ whose image contains $P$ and $P \times 0$ where 0 is the origin in $\mathbb{A}^{r}$. Let $Z^{\prime}=Y \times_{Y \times \mathbb{A}^{r}} Z$, so the induced morphism $Z^{\prime} \rightarrow W$ is a section of a smooth morphism. Applying Artin's approximation to $Z^{\prime}$ and $Y$ yields étale morphisms $Z \rightarrow Y$ and $Z \rightarrow Z^{\prime}$ and a commutative diagram

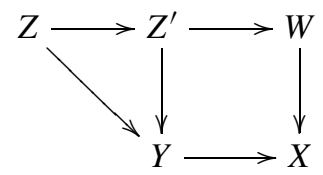

Corollary 2.5 follows from Theorem 2.4 and the following proposition.

PROPOSITION 3.3. Let $\mathrm{BR}$ be a resolution of singularities functor that is functorial for smooth morphisms [Kol, Chapter 3], and let $i: Y \rightarrow X$ be a tubular regular embedding. Let $\tilde{X}=\mathrm{BR}(X)$. Then $i^{*} \tilde{X}$ is a resolution of singularities of $\tilde{X}$. (Here $i^{*} \tilde{X}$ refers to the fiber product $Y \times_{X} \tilde{X}$.)

Proof. First, suppose that $i: Y \rightarrow X$ is a section of a smooth morphism $\pi: X \rightarrow Y$. In this case, by functoriality, $\tilde{X}=\pi^{*} \tilde{Y}$ where $\tilde{Y}=\operatorname{BR}(Y)$. Since $\pi \circ i=1_{Y}, i^{*} \tilde{X}=\tilde{Y}$.

For the general case, observe that if $Y \rightarrow X$ is a regular embedding, then the image of the smooth locus of $Y$ is contained in the smooth locus of $X$. The reason is that any regular embedding with a smooth source must have a smooth target by [EGA4, Thm. 17.12.1].

Thus, $i^{*} Y \rightarrow Y$ is an isomorphism over the smooth locus of $Y$. To finish the proof, we must show that $i^{*} Y$ is smooth. This can be done after étale base change. By hypothesis we have a commutative diagram

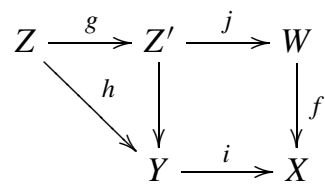


where $j$ is a section of a smooth morphism and $f, g, h$ are étale. Hence, $\tilde{Z}=$ $g^{*} j^{*} f^{*} \tilde{X}$. On the other hand, by commutativity $g^{*} j^{*} f^{*} \tilde{X}=h^{*} i^{*} \tilde{X}$. Thus, $i^{*} \tilde{X}$ is smooth after base change by the étale morphism $h$.

Remark 3.4. A result similar to Proposition 3.3 was proved by Lowengrub [Low].

\subsection{Orbifold Products and Proof of Theorem 2.7}

3.3.1. Definition of the Obstruction Class and Orbifold Product. If $\mathcal{X}$ is a smooth Deligne-Mumford stack with finite stabilizer, then Chen and Ruan [CR] defined an exotic ring structure on the cohomology of the inertia stack $I \mathcal{X}$ called the orbifold product. This product has been studied by many authors and extended to both Chow groups and K-theory. We briefly recall the definition of the orbifold product using the formalism developed in [JKK; EJK1; EJK2].

Denote by $I^{2} \mathcal{X}$ the fiber product $I \mathcal{X} \times \mathcal{X} I \mathcal{X}$. Denote by $e_{1}$ and $e_{2}$ the two projections $I^{2} \mathcal{X} \rightarrow \mathcal{X}$. Since $I^{2} \mathcal{X}$ has the structure as a relative group scheme over $I \mathcal{X}$, there is an additional morphism $\mu: I^{2} \mathcal{X} \rightarrow I \mathcal{X}$ corresponding to the composition in this group.

The orbifold product on $\mathrm{CH}^{*}(I \mathcal{X})$ is defined as follows. Given $\alpha, \beta \in$ $\mathrm{CH}^{*}(I \mathcal{X})$,

$$
\alpha \star \beta=\mu_{*}\left(e_{1}^{*} \alpha \cdot e_{2}^{*} \beta \cdot \mathrm{eu}\left(\mathscr{R}_{\mathcal{X}}\right)\right)
$$

where $\mathscr{R}_{\mathcal{X}}$ is the obstruction bundle and eu denotes its top Chern class. (Note that $I \mathcal{X}$ is in general not equidimensional.) This product preserves the age grading on the Chow groups of $I \mathcal{X}$. (See [JKK] for the definition of the age grading.)

An analogous product can be defined in $K$-theory, where the symbol eu( $\left.\mathscr{R}_{X}\right)$ refers to the $K$-theoretic Euler class $\lambda_{-1}\left(\mathscr{R}_{X}^{*}\right)$.

We denote by $\mathrm{CH}_{\text {orb }}^{*}(\mathcal{X})$ the group $\mathrm{CH}^{*}(I \mathcal{X})$ with the orbifold product and age grading.

If we make the very mild assumption that $\mathcal{X}=[X / G]$ with $G$ a linear algebraic group acting with finite stabilizer on a smooth algebraic space $\mathcal{X}$, then the formalism of [JKK; EJK1; EJK2] can be used to define the class of the obstruction bundle in $K_{0}\left(I^{2} \mathcal{X}\right)$, the Grothendieck group of vector bundles on $I^{2} \mathcal{X}$. To do this, we recall the definition of the logarithmic trace.

Definition 3.5 [EJK1, Definition 4.3]. Let $X$ be an algebraic space with the action of an algebraic group $Z$, and let $V \rightarrow X$ be a $Z$-equivariant vector bundle on $X$. Let $g$ be a finite order automorphism of order $r$ of the fibers of $V \rightarrow X$ such that the action of $g$ commutes with the action of $Z$. Set $L(g)(V)=\sum_{k=1}^{r-1} \frac{k}{r} V_{k} \in$ $K_{0}(Z, X) \otimes \mathbb{Q}$ where $V_{k}$ is the $e^{2 \pi i k / r}$-eigenspaces for the action of $g$. (Here $K_{0}(Z, X)$ denotes the Grothendieck group of $Z$-equivariant vector bundles on $X$.)

REMARK 3.6. Observe that $L(g)(V)=0$ if and only if $g$ acts trivially on the fibers of $V \rightarrow X$. 
Under the assumption that $\mathcal{X}=[X / G], I \mathcal{X}=\left[I_{G} X / G\right]$ and $I^{2} \mathcal{X}=\left[I_{G}^{2} X / G\right]$ where $I_{G} X=\{(g, x) \mid g x=x\}$ and $I_{G}^{2} X=\left\{\left(g_{1}, g_{2}, x\right) \mid g_{1} x=g_{2} x\right\}$. Following [EJK1], $I_{G}^{2} X$ decomposes into a disjoint sum indexed by double conjugacy ${ }^{2}$ classes $\Psi \subset G \times G$. Specifically, $I_{G} X=\bigsqcup_{\Phi} I_{\Phi}$ where

$$
I_{\Phi}=\left\{\left(g_{1}, g_{2}, x\right) \mid g_{1} x=g_{2} x=x,\left(g_{1}, g_{2}\right) \in \Phi\right\} .
$$

If $\left(g_{1}, g_{2}\right) \in \Phi$, then $\left[I_{\Phi} / G\right]=\left[X^{g_{1}, g_{2}} / Z_{G}\left(g_{1}, g_{2}\right)\right]$ where $Z_{G}\left(g_{1}, g_{2}\right)$ is the subgroup of $G$ centralizing $g_{1}$ and $g_{2}$.

On a component $\left[I_{\Phi} / G\right]=\left[X^{g_{1}, g_{2}} / Z_{G}\left(g_{1}, g_{2}\right)\right]$, the class of the obstruction bundle is given as an element of $K_{0}\left(Z_{G}\left(g_{1}, g_{2}\right), X^{g_{1}, g_{2}}\right)$ by the formula [EJK2, Defs. 2.2.3, 2.2.6, 2.3.3]

$$
\begin{aligned}
\operatorname{LR}(\mathbb{T}):= & L\left(g_{1}\right)\left(\left.\mathbb{T}\right|_{X^{g_{1}, g_{2}}}\right)+L\left(g_{2}\right)\left(\left.\mathbb{T}\right|_{X^{g_{1}, g_{2}}}\right) \\
& +L\left(\left(g_{1} g_{2}\right)^{-1}\right)\left(\left.\mathbb{T}\right|_{X^{g_{1}, g_{2}}}\right)-\left.\mathbb{T}\right|_{X^{g_{1}, g_{2}}}+\left(\left.\mathbb{T}\right|_{X^{g_{1}, g_{2}}}\right)^{g_{1}, g_{2}} .
\end{aligned}
$$

Here $\mathbb{T}$ is the class in $K_{0}(G, X)$ corresponding to the tangent bundle of the stack $\mathcal{X}=[X / G]$.

\subsection{Proof of Theorem 2.7}

THEOREM 3.7. Let $\mathcal{Y} \rightarrow \mathcal{X}$ be a strong regular embedding of smooth tame Deligne-Mumford quotient stacks, and let $\mathscr{R}_{X}$ be the class of the obstruction bundle for the orbifold product in $K_{0}\left(I^{2} \mathcal{X}\right)$. Then $\mathscr{R}_{Y}=I^{2} \iota^{*} \mathscr{R}_{X}$ where $\mathscr{R}_{Y}$ is the obstruction bundle for the orbifold product on $\mathcal{Y}$ and $I^{2} \iota: I^{2} \mathcal{Y} \rightarrow I^{2} \mathcal{X}$ is the inclusion.

A necessary ingredient in the proof of Theorem 3.7 is the following property of strong regular embeddings.

Proposition 3.8. Let $\iota: \mathcal{Y} \rightarrow \mathcal{X}$ be a strong regular embedding of tame smooth Deligne-Mumford stacks, and let IY and IX be their respective inertia stacks. Then the normal bundle of IY in I $\mathcal{X}$ is the pullback of the normal bundle of $\mathcal{Y}$ in $\mathcal{X}$. Likewise, the normal bundle to $I^{2} \mathcal{Y}$ in $I^{2} \mathcal{X}$ is the pullback of the normal bundle of $\mathcal{Y}$ in $\mathcal{X}$.

EXAMPLE 3.9. The hypothesis that $\mathcal{Y}$ is smooth is crucial. Let $\mathcal{X}=\left[\mathbb{A}^{2} / \mu_{2}\right]$ where $\mu_{2}$ acts by $-1 \cdot(a, b)=(-a,-b)$. Let $V=V(x y)$, and let $\mathcal{V}=\left[V / \mu_{2}\right]$. Since $V$ is defined by a $\mu_{2}$-invariant function, the embedding $\mathcal{V} \rightarrow \mathcal{X}$ is a strong regular embedding of pure codimension one. The inertia $I \mathcal{X}$ is the disjoint sum $\mathcal{X} \amalg B \mu_{2}$, and the inertia $I \mathcal{Y}$ is the disjoint sum of $\mathcal{Y} \bigsqcup B \mu_{2}$. Thus, the embedding of $I \mathcal{Y}$ in $I \mathcal{X}$ does not have pure codimension one, so the normal bundle if $I \mathcal{Y}$ in $I \mathcal{X}$ cannot be the pullback of the normal bundle of $\mathcal{Y}$ in $\mathcal{X}$.

\footnotetext{
${ }^{2}$ A double conjugacy class is the orbit of a pair $\left(g_{1}, g_{2}\right) \subset G \times G$ under the diagonal action of $G$ by conjugation.
} 
Proof of Proposition 3.8. Since $\mathcal{Y} \rightarrow \mathcal{X}$ is a closed embedding, the diagram

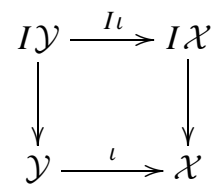

is Cartesian. Moreover, since $\mathcal{Y}$ and $\mathcal{X}$ are smooth, $I \mathcal{Y}$ and $I \mathcal{X}$ are also smooth, so $I \iota$ is also a regular embedding. To check that the normal bundle of $I \mathcal{Y}$ in $I \mathcal{X}$ is the pullback of the normal bundle of $\mathcal{Y}$ and $\mathcal{X}$, it suffices to prove that the two embeddings have the same codimension.

This can be checked after base change by strongly étale morphism. Thus, we can assume that $\mathcal{X}=U / G$, where $U$ is affine and $G$ is a finite group, and $\mathcal{Y}=$ $[V / G]$, where $V$ is a closed subscheme cut out by a $G$-fixed regular sequence. Since $\mathcal{X}=[U / G]$, the inertia stack $I \mathcal{X}$ is the quotient stack $\left[I_{G} U / G\right]$ where $I_{G} X$ is the inertia group for the action of $G$. Since $G$ is finite, $I_{G} U=\bigsqcup_{g \in G} U^{g}$ where $U^{g}$ is the subscheme fixed by $g$. Likewise, $I \mathcal{Y}=\left[I_{G} V / G\right]$ and $I_{G} V=\bigsqcup_{g \in G} V^{g}$.

Thus, it suffices to check that $V \hookrightarrow U$ is a $G$-equivariant embedding of affine schemes of pure codimension $d$ and $V$ is defined by a $G$-fixed regular sequence. Then for any $g \in G$, the codimension of $V^{g}$ in $U^{g}$ is also $d$. Let $y \in V^{g}$ be a point, and let $G_{y}$ be the stabilizer of $y$, which necessarily contains $g$. The argument used in the proof of Theorem 2.2 shows that the complete local ring $\widehat{\mathcal{O}}_{y, U}$ is isomorphic as a $G_{y}$-module to $\widehat{\mathcal{O}}_{y, V}\left[\left[T_{1}, \ldots, T_{d}\right]\right]$ where $G_{y}$ acts trivially on the $T_{i}$. Hence,

$$
\widehat{\mathcal{O}}_{y, U}=\widehat{\mathcal{O}}_{y, U}^{\langle g\rangle}=\mathcal{O}_{y, V}^{\langle g\rangle}\left[\left[T_{1}, \ldots, T_{d}\right]\right],
$$

so the codimension is preserved.

A similar argument shows that the normal bundle of $I^{2} \mathcal{Y}$ in $I^{2} \mathcal{X}$ is also the pullback of the normal bundle to $I \mathcal{Y}$ in $I \mathcal{X}$.

Proof of Theorem 3.7. If $\mathcal{Y} \hookrightarrow \mathcal{X}$ is a strong regular embedding, then by Proposition 3.8 we have the following identity in $K_{0}\left(Z_{G}\left(g_{1}, g_{2}\right),\left(Y^{g_{1}, g_{2}}\right)\right)$ :

$$
\left.\mathbb{T} \mathcal{X}\right|_{Y^{g_{1}, g_{2}}}=\left.\mathbb{T} \mathcal{Y}\right|_{Y^{g_{1}, g_{2}}}+\left.N\right|_{Y^{g_{1}, g_{2}}}
$$

where $N$ is the normal bundle of $\mathcal{Y}$ in $\mathcal{X}$. Moreover, since $\iota$ is a strong regular embedding, the fibers of $\left.N\right|_{Y^{g_{1}}, g_{2}}$ are trivial modules for the action of the group generated by $g_{1}, g_{2}$. Hence, $L\left(g_{1}\right)(N)=L\left(g_{2}\right)(N)=L\left(\left(g_{1} g_{2}\right)^{-1}\right)(N)=0$. Also, since $g_{1}, g_{2}$ act trivially on $N, N=N^{g_{1}, g_{2}}$. Substituting into formula (2), we see that $\left.L T(\mathbb{T} \mathcal{X})\right|_{Y_{1} g_{2}}=L T(\mathbb{T} \mathcal{Y})$, which proves the proposition.

Proof of Theorem 2.7. To prove the theorem, we must show the following identity for any $\alpha, \beta \in \mathrm{CH}^{*}(I \mathcal{X})$ :

$$
\mu_{*}\left(e_{1}^{*}(I \iota)^{*}(\alpha) \cdot e_{2}^{*}(I \iota)^{*}(\beta) \cdot \operatorname{eu}(\mathscr{R} \mathcal{Y})\right)=\left(I^{2} \iota\right)^{*}\left(\mu_{*}\left(e_{1}^{*} \alpha \cdot e_{2}^{*} \beta \cdot \operatorname{eu}\left(\mathscr{R}_{\mathcal{X}}\right)\right) .\right.
$$

By Theorem $3.7, \mathscr{R}_{\mathcal{Y}}=\left(I^{2} \iota\right)^{*} \mathscr{R} \mathcal{X}$. By Proposition 3.8 the normal bundle of $I^{2} \mathcal{Y}$ in $I^{2} \mathcal{X}$ is the pullback of the normal bundle to $I \mathcal{Y}$ in $I \mathcal{X}$. It follows [Ful, Thm. 6.2(b, c) ] that $e_{i}^{*} \circ(I \iota)^{*}=\left(I^{2} \iota\right) \circ e_{i}^{*}$ as morphisms $\mathrm{CH}^{*}(I \mathcal{X}) \rightarrow \mathrm{CH}^{*}\left(I^{2} \mathcal{Y}\right)$. 
Applying [Ful, Thm. 6.2(a, b)] also implies that $\mu_{*} \circ\left(I^{2} \iota\right)^{*}=(I \iota)^{*} \circ \mu_{*}$ as a morphism $\mathrm{CH}^{*}\left(I^{2} \mathcal{X}\right) \rightarrow \mathrm{CH}^{*}(I \mathcal{Y})$.

Substituting these identities into equation (3) yields the theorem.

\subsection{Examples}

Let $\mathcal{X}=\left[\mathbb{A}^{3} / \mu_{3}\right]$ where the generator $\omega$ of $\mu_{3}$ acts by $\omega(a, b, c)=\left(a, \omega b, \omega^{2} c\right)$. Since $\mathbb{A}^{3}$ is a representation of $\mu_{3}, \mathrm{CH}^{*}(\mathcal{X})=\mathrm{CH}^{*}\left(B \mu_{3}\right)=\mathbb{Z}[t] / 3 t$. The inertia $I \mathcal{X}$ has three components indexed by $\mu_{3}$. The identity section $I_{1}$ is isomorphic to $\mathcal{X}$, and the components $I_{\omega}$ and $I_{\omega^{2}}$ are both isomorphic to $\mathbb{A}^{1} \times B \mu_{3}$, which is identified with the quotient $\left[\{(a, 0,0)\} / B \mu_{3}\right]$. The Chow groups $\mathrm{CH}^{*}(I \mathcal{X})$ have a natural $\mathrm{CH}^{*}\left(B \mu_{3}\right)$-module structure, which is preserved by the orbifold product, so we write $\mathrm{CH}^{*}(I \mathcal{X})=\bigoplus_{m \in \mu_{3}} \mathbb{Z}[t] /(3 t)$.

Since $\mu_{3}$ is abelian, there are nine double conjugacy classes, and $I^{2} \mathcal{X}$ has nine components indexed by $\mu_{3} \times \mu_{3}$. For each pair $\left(m_{1}, m_{2}\right) \in \mu_{3} \times \mu_{3}$, let $\mathscr{R}\left(m_{1}, m_{2}\right)$ be the restriction of the obstruction bundle to the component $I_{m_{1}, m_{2}}^{2}=$ $\left[\left(\mathbb{A}^{3}\right)^{m_{1}, m_{2}} / \mu_{3}\right]$. Let $\xi$ be the defining representation of $\mu_{3}$. Then the tangent bundle $\mathbb{T}$ of $\mathcal{X}$ corresponds to the representation $1+\chi+\chi^{2}$ where 1 denotes the trivial representation. Using the formula of [EJK1], we obtain $\mathscr{R}(\omega, \omega)=\chi^{2}$, $\mathscr{R}\left(\omega^{2}, \omega^{2}\right)=\chi$, and $\mathscr{R}\left(m_{1}, m_{2}\right)=0$ for all other pairs $\left(m_{1}, m_{2}\right) \in \mu_{3} \times \mu_{3}$.

If we denote by $l_{m}$ with $m \in \mu_{3}$ the generator of the $\mathbb{Z}[t] /(3 t)$-module $\mathrm{CH}^{*}\left(I_{m}\right)$, then we obtain the following identities for the orbifold product:

$$
\begin{aligned}
l_{\omega} \star l_{\omega} & =2 t l_{\omega}, \\
l_{\omega} \star l_{\omega^{2}} & =2 t^{2} l_{1}, \\
l_{\omega^{2}} \star l_{\omega^{2}} & =t l_{\omega},
\end{aligned}
$$

and $l_{1}$ acts as the identity. Hence,

$$
\mathrm{CH}_{\text {orb }}^{*}(I \mathcal{X})=\mathbb{Z}\left[t, l_{\omega}, l_{\omega^{2}}\right] /\left(3 t, l_{\omega}^{2}-2 t l_{\omega}, l_{\omega} l_{\omega^{2}}-2 t^{2}, l_{\omega^{2}}^{2}-t l_{\omega^{2}}\right) .
$$

We will now consider various substacks of $\mathcal{X}$ and compare orbifold Chow rings. To start, let $\mathcal{Y}=\left[\mathbb{A}^{2} / \mu_{3}\right]$ where $\omega \cdot(b, c)=\left(\omega b, \omega^{2} c\right)$. The map $\mathbb{A}^{2} \rightarrow \mathbb{A}^{3}$, $(b, c) \mapsto(0, b, c)$ induces a strong regular embedding of stacks $\mathcal{Y} \rightarrow \mathcal{X}$ since the ideal of $\mathbb{A}^{2}$ in $\mathbb{A}^{3}$ is generated by the $\mu_{3}$-fixed function $x$. Again, $\mathrm{CH}^{*}(I \mathcal{Y})$ is isomorphic to the $\bigoplus_{m \in \mu_{3}} \mathbb{Z}[t] /(3 t)$, and the pullback induced by the inclusion $I \mathcal{Y} \rightarrow I \mathcal{X}$ maps $l_{\omega}$ to $l_{\omega}$ and $l_{\omega^{2}}$ to $l_{\omega^{2}}$, and direct calculation shows that the pullback induces an isomorphism $\mathrm{CH}_{\text {orb }}^{*}(\mathcal{Y}) \rightarrow \mathrm{CH}_{\text {orb }}^{*}(\mathcal{X})$.

Now let $\mathcal{Y}$ be the substack $\left[\mathbb{A}^{2} / \mu_{3}\right]$ where $\omega \cdot(a, b)=(a, \omega b)$. The map $\mathbb{A}^{2} \rightarrow \mathbb{A}^{3},(a, b) \mapsto(a, b, 0)$, induces an embedding $\mathcal{Y} \rightarrow \mathcal{X}$, but since the defining equation of $\mathbb{A}^{2}$ in $\mathbb{A}^{3}$ is not $\mu_{3}$-invariant, this is not a strong regular embedding. Indeed, a direct calculation shows that $\mathscr{R}(\omega, \omega)=0$, so that $\mathscr{R} \mathcal{Y}$ is not the pullback of $\mathscr{R}_{\mathcal{X}}$. Again, $\mathrm{CH}^{*}(I \mathcal{Y})$ is isomorphic to $\bigoplus_{m \in \mu_{3}} \mathbb{Z}[t] /(3 t)$, and the pullback induced by the inclusion $\mathcal{Y} \rightarrow \mathcal{X}$ maps $l_{m}$ to $l_{m}$. However, because $\mathscr{R}_{\omega, \omega}=0$, the orbifold Chow ring has the presentation

$$
\mathrm{CH}_{\mathrm{orb}}^{*}(I \mathcal{Y})=\mathbb{Z}\left[t, l_{1}, l_{2}\right] /\left(3 t, l_{\omega^{2}}-l_{\omega}, l_{\omega} l_{\omega^{2}}-2 t^{2}, l_{\omega^{2}} l_{\omega^{2}}-t l_{\omega^{2}}\right),
$$


and we see that the map that sends the generator $l_{m}$ of $\mathrm{CH}^{*}\left(I \mathcal{X}_{m}\right)$ to the generator $l_{m}$ of $\mathrm{CH}^{*}\left(I \mathcal{Y}_{m}\right)$ is not a homomorphism of orbifold Chow rings.

Finally, let $\mathcal{Y}=\left[Z / \mu_{3}\right]$ where $Z=V(y z-1) \subset \mathbb{A}^{3}$. Since the equation $y z-1$ is $\mu_{3}$-fixed, the map $\mathcal{Y} \rightarrow \mathcal{X}$ is a strong regular embedding. Since $\mu_{3}$ acts freely on $Z$, on the quotient stack $\mathcal{Y}$ is represented by the scheme $\mathbb{A}^{1} \times \mathbb{G}_{m}$. Thus, $I \mathcal{Y}=$ $\mathcal{Y}$, and so $(I \mathcal{Y})_{\omega}$ and $(I \mathcal{Y})_{\omega^{2}}$ are both empty. Thus, $\mathrm{CH}^{*}(I \mathcal{Y})=\mathrm{CH}^{*}\left(\mathbb{A}^{1} \times \mathbb{G}_{m}\right)=$ $\mathbb{Z}$, and the orbifold product is trivial. The pullback induced by the inclusion $\mathcal{Y} \rightarrow$ $\mathcal{X}$ maps $l_{\omega}$ and $l_{\omega^{2}}$ to 0 , and the map on orbifold Chow rings is the homomorphism

$$
\mathbb{Z}\left[t, l_{\omega}, l_{\omega}^{2}\right] /\left(3 t, l_{\omega}^{2}-2 t l_{\omega}, l_{\omega} l_{\omega^{2}}-2 t^{2}, l_{\omega^{2}}^{2}-t l_{\omega^{2}}\right) \rightarrow \mathbb{Z}
$$

given by setting $t, l_{\omega}, l_{\omega^{2}}$ to be equal to 0 .

\section{Application: The Generalized Euler Sequence and Cotangent Bundle Stacks}

Let $T$ be a torus acting on a smooth variety $X$ defined over a field $k$ so that the quotient stack $\mathcal{X}=[X / T]$ is a tame Deligne-Mumford stack.

In this section we consider two natural quotient stacks that arise from this data, namely $T^{*} \mathcal{X}$ and $\left[T^{*} X / T\right]$. The first stack is intrinsic to $\mathcal{X}$, whereas the latter depends on the presentation of $\mathcal{X}$ as a quotient stack. There is an exact sequence of vector bundles on $\mathcal{X}$

$$
0 \rightarrow T^{*} \mathcal{X} \rightarrow\left[T^{*} X / T\right] \rightarrow\left[X \times \operatorname{Lie}(T)^{*} / T\right] \rightarrow 0 .
$$

Since $T$ is a torus, $\operatorname{Lie}(T)$ is a trivial $T$-module, and the last bundle in the sequence is the trivial bundle $[X / T] \times \operatorname{Lie}(T)$. We call (4) the generalized Euler sequence because, when $X=\mathbb{A}^{n+1} \backslash\{0\}$ and $T=\mathbb{G}_{m}$ acts with weights all equal to one, then $\mathcal{X}=[X / T]=\mathbb{P}^{n}$, and (4) is the usual Euler sequence for the cotangent bundle of $\mathbb{P}^{n}$.

Our goal is to understand the relationship between the stacks $T^{*} \mathcal{X}$ and $\left[T^{*} X / T\right]$. Since both stacks are vector bundles over $\mathcal{X}$, the inclusion $i: T^{*} \mathcal{X} \rightarrow$ $\left[T^{*} X / T\right]$ induces a pullback isomorphism $i^{*}: \mathrm{CH}^{*}\left(\left[T^{*} X / T\right]\right) \rightarrow \mathrm{CH}^{*}(\mathcal{X})$.

However, stronger results hold. Let $\mathbf{M}$ be the coarse space of $T^{*} \mathcal{X}$, and let $\mathbf{N}$ be the coarse space of $\left[T^{*} X / T\right]$. By Sumihiro's theorem [Sum] the action of a torus on a normal variety is locally linearizable in the Zariski topology. This implies that the coarse spaces $\mathbf{M}, \mathbf{N}$, and $\mathbf{X}$ are all $k$-varieties.

There is a commutative triangle of morphisms of schemes:

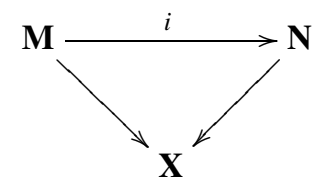

Note that in general $\mathbf{M}$ and $\mathbf{N}$ are not vector bundles over $\mathbf{X}$.

Proposition 4.1. The inclusion $\iota: T^{*} \mathcal{X} \rightarrow\left[T^{*} X / T\right]$ is a strong regular embedding, so the inclusion $\mathbf{M} \rightarrow \mathbf{N}$ is a regular embedding that is étale locally isomorphic to a section of a smooth morphism. 
Proof. Observe that the sequence $T^{*} \mathcal{X} \rightarrow\left[T^{*} X / T\right] \rightarrow\left[X \times \operatorname{Lie}(T)^{*} / T\right]$ is exact and $\operatorname{Lie}(T)^{*}$ is a trivial $T$-module (because $T$ is diagonalizable). Hence, the normal bundle to $\iota$ is the trivial bundle $T^{*} \mathcal{X} \times \operatorname{Lie}(T)^{*}$, so $\iota$ is a strong regular embedding.

COROLlary 4.2. If $\tilde{\mathbf{N}}$ is a canonical (functorial) resolution of singularities of $\mathbf{N}$, then $\tilde{\mathbf{M}}:=\mathbf{M} \times_{\mathbf{N}} \tilde{\mathbf{N}}$ is a resolution of singularities as well.

The main result of this section is the following result about the Chow groups of $\mathbf{M}$ and $\mathbf{N}$.

THEOREM 4.3. The pullback $\iota^{*}: \mathrm{CH}_{*}(\mathbf{N}) \rightarrow \mathrm{CH}_{*-\operatorname{dim} T}(\mathbf{M})$ is an isomorphism of integral Chow groups.

Theorem 4.3 follows from a more general result about strong regular embeddings of vector bundles on not-necessarily smooth quotient stacks.

Let $T$ be a diagonalizable group (as opposed to a torus) acting on a reduced scheme $X$ such that the quotient $\mathcal{X}=[X / T]$ is a tame Deligne-Mumford stack.

Let

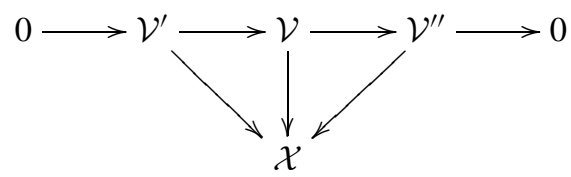

be a short exact sequence of vector bundles on $\mathcal{X}$ such that there is a stratification of $\mathcal{X}$ on which $\mathcal{V}^{\prime \prime}$ is trivial, so the inclusion $\mathcal{V}^{\prime} \rightarrow \mathcal{V}$ is a strong regular embedding by Theorem 2.2. Denote by $\mathbf{V}$ and $\mathbf{V}^{\prime}$ the coarse moduli spaces of $\mathcal{V}$ and $\mathcal{V}^{\prime}$, respectively, and let $i: \mathbf{V}^{\prime} \rightarrow \mathbf{V}$ be the inclusion.

THEOREM 4.4. If $\mathbf{V}$ is a scheme, then the pullback on (higher) Chow groups $i^{*}: \mathrm{CH}_{*}(\mathbf{V}, i) \rightarrow \mathrm{CH}_{*-d}\left(\mathbf{V}^{\prime}, i\right)$ is an isomorphism with integer coefficients.

REMARK 4.5. Note that since $T$ is only assumed diagonalizable, it is not necessarily connected, so the coarse spaces for the quotient stacks are not a priori schemes. However, the assumption that $\mathbf{V}$ is a scheme is required for the proof because we use the localization theorem for higher Chow groups. If we knew that this sequence was also valid for algebraic spaces, then the theorem would go through without this hypothesis.

We first prove a special case of Theorem 4.4.

Lemma 4.6. The conclusion of Theorem 4.4 holds for classifying stacks BH where $H$ is a finite, linearly reductive group.

Proof. A vector bundle on $B H$ is a stack $\mathcal{V}=[V / H]$ where $V$ is a linear representation of $H$. The coarse space of $\mathcal{V}$ is the quotient scheme $V / H=$ $\operatorname{Spec} \mathcal{O}(V)^{H}$. 
By hypothesis we are given a short exact sequence

$$
0 \rightarrow V^{\prime} \rightarrow V \rightarrow V^{\prime \prime} \rightarrow 0
$$

of $H$-modules such that $V^{\prime \prime}$ is trivial.

Since $H$ is linearly reductive, any short exact sequence of $H$-modules splits, so $V=V^{\prime} \oplus V^{\prime \prime}$, and the inclusion $V^{\prime} \rightarrow V=V^{\prime} \oplus V^{\prime \prime}$ is the 0 -section of the projection $V^{\prime} \oplus V^{\prime \prime} \rightarrow V^{\prime}$. Moreover, since $H$ acts trivially on $V^{\prime \prime}, V / H=V^{\prime} / H \times V^{\prime \prime}$, and the inclusion of quotients $V^{\prime} / H \rightarrow V / H$ is the inclusion of the 0-section of the trivial vector bundle $V^{\prime} / H \times V \rightarrow V^{\prime} / H$. Since the pullback along the 0 -section of a vector bundle induces isomorphisms of (higher) Chow groups, the lemma follows.

Lemma 4.7. If $\mathcal{X}=[X / T]$ is a reduced Deligne-Mumford quotient stack with $T$ a diagonalizable group, then there is a dense open substack $\mathcal{U} \subset \mathcal{X}$ such that $\mathcal{U}$ is isomorphic to $\mathrm{BH} \times W$ where $H$ is a finite diagonalizable group and $W$ is affine.

Proof. Embedding $T$ into a torus $T^{\prime}$, the stack $\mathcal{X}$ can be presented as the quotient $\left[\left(X \times{ }_{T} T^{\prime}\right) / T^{\prime}\right]$. Since $T$ and $T^{\prime}$ are assumed to be smooth, $X \times_{T} T^{\prime}$ is also reduced. Replacing $T$ with $T^{\prime}$, we may assume that it is a torus. By [Tho, Lemma 4.3] there is a dense $T$-invariant open subspace of $X$ that is a separated scheme. Replacing $\mathcal{X}$ by this open subset, we may assume that $X$ is a reduced, separated scheme.

By [Tho, Prop. 4.10] there are an affine open subspace $U \subset X$ and a diagonalizable subgroup $T^{\prime} \subset T$ with quotient torus $T^{\prime \prime}$ such that $T$ acts via the homomorphism $T \rightarrow T^{\prime \prime}$ and $T^{\prime \prime}$ acts freely on $U$. If we let $W=U / T$, then $U$ is $T$-equivariantly isomorphic to $T / T^{\prime} \times W$. Since $[U / T]$ is Deligne-Mumford, it follows that $H=T^{\prime}$ is a finite group, and so we see that $[U / T]$ is equivalent to $B H \times W$.

Let $\mathcal{V}$ be a vector bundle on $\mathcal{X}$, and let $\mathcal{U}$ be an open substack. Let $\mathcal{V}_{\mathcal{U}}$ denote the restriction of $\mathcal{V}$ to a vector bundle on $\mathcal{U}$, and let $\mathbf{V}_{\mathcal{U}}$ be its coarse moduli space.

LEMMA 4.8. Let $\mathcal{V}^{\prime} \rightarrow \mathcal{V} \rightarrow \mathcal{V}^{\prime \prime}$ be an exact sequence of vector bundles on $\mathcal{X}=[X / T]$ satisfying the hypothesis of the theorem. Then there is a dense open substack $\mathcal{U} \subset \mathcal{X}$ such that the pullbacks $i_{\mathcal{U}}^{*}: \mathrm{CH}_{*}\left(\mathbf{V}_{\mathcal{U}}\right) \rightarrow \mathrm{CH}_{*}\left(\mathbf{V}_{\mathcal{U}}^{\prime}\right)$ is an isomorphism. (Here $i_{\mathcal{U}}: \mathbf{V}_{\mathcal{U}}^{\prime} \rightarrow \mathbf{V}_{\mathcal{U}}$ is the inclusion of coarse spaces.)

Proof. By Lemma 4.7 there is a dense open substack $\mathcal{U} \subset \mathcal{X}$ isomorphic to $B H \times$ $W$ where $H$ is a finite diagonalizable group and $W$ is affine. The restriction of a vector bundle $\mathcal{V}$ to $U$ is a sum of vector bundles of the form $[V / H] \otimes \mathcal{E}$ where $V$ is a representation of $H$ and $\mathcal{E}$ is a vector bundle on $W$. Therefore, given an exact sequence $\mathcal{V}^{\prime} \rightarrow \mathcal{V} \rightarrow \mathcal{V}^{\prime \prime}$, we can shrink $W$ and hence $\mathcal{U}$ so that each of these bundles restricted to $\mathcal{U}$ is of the form $V \otimes \mathcal{O}_{W}$. We can then repeat the argument of Lemma 4.6.

Lemma 4.9. Let $\mathcal{X}$ be a tame Deligne-Mumford stack, and let $\mathcal{U}$ be an open set with complement $\mathcal{Z}$ (with the reduced induced substack structure). Let $\mathbf{X}, \mathbf{U}$, and 
$\mathbf{Z}$ be the coarse spaces of $\mathbf{X}, \mathbf{U}$, and $\mathbf{Z}$, respectively. Then the inclusion $\mathcal{Z} \rightarrow \mathcal{X}$ (resp. $\mathcal{U} \rightarrow \mathcal{X}$ ) induces a closed (resp. open) immersion $\mathbf{Z} \rightarrow \mathbf{X}($ resp. $\mathbf{U} \rightarrow \mathbf{X})$, and $\mathbf{U}=\mathbf{X} \backslash \mathbf{Z}$.

Proof. Again using the structure theorem for coarse moduli spaces, we can reduce to the case that $\mathcal{X}=[\operatorname{Spec} A / G]$ for some finite group $G$. Then $\mathcal{U}=[W / G]$ where $W$ is a $G$-invariant open set. Let $Y=\operatorname{Spec} A \backslash W$. The coarse moduli space of $\mathcal{U}$ is $\pi(W)$ where $\pi: \operatorname{Spec} A \rightarrow \operatorname{Spec} A^{G}$ is the quotient map. Likewise, the coarse moduli space of $\mathcal{Z}$ is $\pi(Y)$.

Since the morphism $\pi$ is a geometric quotient, its geometric fibers are $G$-orbits of geometric points. It follows that $\pi(W)$ is open in $X=\operatorname{Spec} A^{G}$ and equal to $\pi(\operatorname{Spec} A) \backslash \pi(Z)$.

Proof of Theorem 4.4. Observe that if $\mathcal{V}$ is a vector bundle on $\mathcal{X}$ and $\mathcal{U} \subset \mathcal{X}$ is an open substack, then the complement of $\left.\mathcal{V}\right|_{\mathcal{U}}$ (with its reduced induced stack structure) is $\mathcal{V}_{\mathcal{Z}}$ where $\mathcal{Z}$ is the complement of $\mathcal{U}$ with its reduced induced stack structure. Hence, by Lemma 4.9 the complement of the coarse space $\mathbf{V}_{\mathcal{U}}$ is the coarse space $\mathbf{V}_{\mathcal{Z}}$.

Now by Lemma 4.8 there is an open substack $\mathcal{U} \subset \mathcal{V}$ such that the inclusion $\mathbf{V}_{\mathcal{U}}^{\prime} \rightarrow \mathbf{V}_{\mathcal{U}}$ induces a pullback isomorphism of (higher) Chow groups $\mathrm{CH}^{d}\left(\mathbf{V}_{\mathcal{U}}^{\prime}, i\right) \rightarrow \mathrm{CH}^{d}\left(\mathbf{V}_{\mathcal{U}}, i\right)$ for every $d, i$. By Noetherian induction we may assume that the inclusion $\mathbf{V}_{\mathcal{Z}}^{\prime} \rightarrow \mathbf{V}_{\mathcal{Z}}$ also induces a pullback isomorphism of (higher) Chow groups. The theorem follows by applying the localization exact sequence for higher Chow groups (1).

Proof of Corollary 2.8. By Theorem 2.7 we know that the pullback $(I \iota)^{*}$ : $\mathrm{CH}^{*}(I(T \mathcal{X})) \rightarrow I\left(\left[T^{*} X / G\right]\right)$ commutes with the orbifold product. Thus, it suffices to show that $(I \iota)^{*}$ is an isomorphism of Abelian groups. This follows because both $T^{*} \mathcal{X}$ and $\left[T^{*} X / G\right]$ are vector bundles over $\mathcal{X}=[X / G]$, so $I T^{*} \mathcal{X}$ and $I\left[T^{*} X / G\right]$ are both vector bundles over $I \mathcal{X}$. Hence, the pullback $I \iota^{*}$ is an isomorphism.

REMARK 4.10. The methods used to prove Theorem 4.3 and Theorem 4.4 yield analogous isomorphisms for the $K$-theory of coherent sheaves. Since $K$-theory is naturally defined for schemes over an arbitrary base, we do not need to assume that the base scheme is a field. Also, there is a localization long exact sequence for the higher $K$-theory of algebraic spaces, so we can obtain the $K$-theory result in more generality. In particular, we may assume that $X$ is a smooth algebraic space defined over a Noetherian scheme $S$ and that $T$ is diagonalizable, that is, isomorphic to a closed subgroup of a torus.

\section{Application: Hypertoric Stacks and Lawrence Toric Stacks}

For simplicity of exposition (see Remark 5.1), we work over an algebraically closed field of characteristic 0 . 
A natural algebraic way to construct hypertoric varieties is via geometric invariant theory (GIT) as follows.

Fix integers $d, n$ with $n \geq d$ and let $V$ be an $n$-dimensional representation of the rank $d$ torus $T$. Choosing a diagonalizing basis for the action of $T_{d}$, let $A=\left(a_{i j}\right) \in \mathbb{Z}^{d \times n}$ be the matrix of weights for the action of $d$. We denote by $a_{k}$ the $k$ th column vector of $A$. We assume that $A$ has maximal rank over $\mathbb{Q}$, which is equivalent to assuming that the generic stabilizer is finite.

There is a natural action of $T$ on $V \times V^{*}$, and with an appropriate choice of basis, the weight matrix for this action is $A^{ \pm}=\left(a_{1}, \ldots, a_{n}-a_{1}, \ldots,-a_{n}\right) \in$ $\mathbb{Z}^{d \times 2 n}$.

If $\theta \in \mathbb{Z}^{d}$ is a character of $T$, then we can consider the $\theta$-stable and semistable loci in $V \times V^{*}$. For generic choice of $\theta,\left(V \times V^{*}\right)^{s}=\left(V \times V^{*}\right)^{s s}$. The quotient stack $\mathcal{X}\left(A^{ \pm}, \theta\right):=\left[\left(V \times V^{*}\right)^{s} / T\right]$ is called a Lawrence toric stack. The GIT quotient $\mathbf{X}\left(A^{ \pm}, \theta\right):=\left(V \times V^{*}\right)_{\theta} / / T$ is the coarse moduli space of $\mathcal{X}\left(A^{ \pm}, \theta\right)$ and is called a Lawrence toric variety. Note that the coordinate ring of $V \times V^{*}$ contains invariant elements, so the GIT quotient $\mathbf{X}\left(A^{ \pm}, \theta\right)$ is not projective. However, it is semiprojective, meaning that it is projective over $\operatorname{Spec} \operatorname{Sym}\left(\left(V \times V^{*}\right)\right)^{T}$.

The representation $V \times V^{*}$ has a natural $T$-invariant algebraic symplectic pairing $\mu:\left(V \times V^{*}\right) \rightarrow \operatorname{Lie}\left(T_{d}\right)^{*}=\mathbb{C}^{d}$. If we choose coordinates $\left(x_{1}, \ldots, x_{n}\right)$ on $V$ and dual coordinates $\left(y_{1}, \ldots, y_{n}\right)$ on $V^{*}$ so that $T_{d}$ acts on $x_{i}$ with weight $a_{i}$ and on $y_{i}$ with weight $-a_{i}$, then $\mu$ is given by the formula

$$
\left(x_{1}, \ldots, x_{n}, y_{1}, \ldots, y_{n}\right) \mapsto\left(\mu_{1}, \ldots, \mu_{n}\right),
$$

where $\mu_{i}=\sum_{j} a_{i j} x_{j} y_{j}$.

The quotient $\mathbf{Y}(A, \theta)=\mu^{-1}(0) / /{ }_{\theta} T$ is the associated hypertoric variety. Following Jiang and Tseng [JT2], we refer to the quotient stack

$$
\mathcal{Y}(A, \theta):=\left[\left(\mu^{-1}(0) \cap\left(V \times V^{*}\right)^{s}\right) / T\right]
$$

as a hypertoric stack. The variety $\mathbf{Y}(A, \theta)$ is the coarse space of $\mathcal{Y}(A, \theta)$.

REMARK 5.1. We can also consider hypertoric stacks defined over an algebraically closed field of characteristic $p$, provided we assume that the weights $a_{i}$ are all coprime to $p$. This assumption ensures that our hypertoric stacks are tame.

EXAMPLE 5.2. If $A=\left(a_{0}, \ldots, a_{n}\right)$ is a $1 \times(n+1)$ matrix with all $a_{i}$ positive, then we denote by $\mathbb{P}\left(a_{0}, \ldots, a_{n}\right)$ the quotient stack $\left[\mathbb{A}^{n+1} \backslash\{0\} / \mathbb{G}_{m}\right]$ where $\mathbb{G}_{m}$ acts with weights $\left(a_{0}, \ldots, a_{n}\right)$. If $\theta$ is positive, then $\mathcal{Y}(A, \theta)=T^{*} \mathbb{P}\left(a_{0}, \ldots, a_{n}\right)$, and the Lawrence toric stack $\mathcal{X}\left(A^{ \pm}, \theta\right)$ equals $\left[T^{*}\left(\mathbb{A}^{n+1} \backslash\{0\}\right) / T\right]$.

THEOREM 5.3. The stack $\mathcal{X}\left(A^{ \pm}, \theta\right)$ has a Zariski open cover by open sets $\mathcal{U}$, each isomorphic to $(\mathcal{Y}(A, \theta) \cap \mathcal{U}) \times \mathbb{A}^{d}$, such that, under this isomorphism, c corresponds to a section of the projection $\mathcal{U} \rightarrow(\mathcal{X}(A, \theta) \cap \mathcal{U})$.

In particular, the inclusion $\iota: \mathcal{Y}(A, \theta) \hookrightarrow \mathcal{X}\left(A^{ \pm}, \theta\right)$ is a strong regular embedding of smooth Deligne-Mumford stacks. 
Applying Corollary 2.5, we obtain as a corollary the following result about resolutions of singularities.

Corollary 5.4. Let $\tilde{\mathbf{X}} \rightarrow \mathbf{X}\left(A^{ \pm}, \theta\right)$ be a canonical resolution of singularities of the Lawrence toric variety $\mathbf{X}\left(A^{ \pm}, \theta\right)$ and set $\tilde{\mathbf{X}}:=\mathbf{Y}(A, \theta) \times_{\mathbf{X}\left(\mathbb{A}^{ \pm, \theta}\right)} \tilde{\mathbf{X}}$. Then $\tilde{\mathbf{Y}} \rightarrow \mathbf{Y}(A, \theta)$ is a resolution of singularities of the hypertoric variety $\mathbf{Y}(A, \theta)$.

As in the case of cotangent bundles, we also have a result about integral Chow groups and Chow rings as well.

Theorem 5.5. The pullback $\iota^{*}: \mathrm{CH}^{*}\left(\mathcal{X}\left(A^{ \pm}, \theta\right)\right) \rightarrow \mathrm{CH}^{*}(\mathcal{Y}(A, \theta))$ is an isomorphism of integral Chow rings of Deligne-Mumford stacks.

Also, if $\mathbf{Y}(A, \theta) \stackrel{i}{\hookrightarrow} \mathbf{X}\left(A^{ \pm}, \theta\right)$ is the inclusion of a hypertoric variety into the corresponding Lawrence toric variety, then the pullback $i^{*}: \mathrm{CH}_{k}\left(\mathbf{X}\left(A^{ \pm}, \theta\right)\right) \rightarrow$ $\mathrm{CH}_{k-d}(\mathbf{Y}(A, \theta))$ is an isomorphism of integral Chow rings.

The analogous statements also hold for the $K$-theory of coherent sheaves.

Proof of Theorem 5.3. By [HS, Cor. 4.4] a point is $\theta$-stable if it lies in the complement of the vanishing of the irrelevant ideal

$$
B_{\theta}=\left\langle\prod_{\sigma(C, \theta)}: C \text { any column basis of } A\right\rangle,
$$

where $\sigma(C, \theta)$ is defined as follows: If $C=\left\{a_{i 1}, \ldots, a_{i d}\right\}$, then there are unique nonzero $^{3}$ rational numbers $\lambda_{1}, \ldots, \lambda_{d}$ such that

$$
\lambda_{1} a_{i 1}+\lambda_{2} a_{i 2}+\cdots+\lambda_{d} a_{i d}=\theta
$$

and

$$
\sigma(C, \theta)=\left\{x_{i_{j}}: \lambda_{j}>0\right\} \cup\left\{y_{i_{l}}: \lambda_{l}<0\right\} .
$$

Each set of indices in $\sigma(C, \theta)$ corresponds to a maximal cone in the fan of the toric stack $\mathcal{X}\left(A^{ \pm}, \theta\right)$. Let $U_{\sigma(C, \theta)} \subset V \times V^{*}$ be the principal open set corresponding to the monomial $x_{\sigma(C, \theta)}=\prod_{\sigma(C, \theta)} x_{i_{j}} y_{i_{l}}$, and let $\mathcal{U}_{\sigma(C, \theta)}=\left[U_{\sigma(C, \theta)} / T\right]$. By construction the $U_{\sigma(C, \theta)}$ form an open cover of $\left(V \times V^{*}\right)^{S}$. Theorem 5.3 then follows from the following proposition.

Proposition 5.6. Let $\sigma=\sigma(C, \theta)$ for some fixed subset $C$ of the columns of $A$. If $W \subset U_{\sigma}$ is a $\mathbb{G}_{m}^{2 n}$-invariant irreducible closed subset transverse to $\mu^{-1}(0)$, then $W$ is $T$-equivariantly isomorphic to $\left(\mu^{-1}(0) \cap W\right) \times \mathbb{A}^{d}$ where $T$ acts trivially on $\mathbb{A}^{d}$. Under this isomorphism, the inclusion $W \cap \mu^{-1}(0) \hookrightarrow W$ corresponds to a section of the projection $\left(\mu^{-1}(0) \cap V\right) \times \mathbb{A}^{d} \rightarrow \mu^{-1}(0) \cap V$.

\footnotetext{
${ }^{3}$ The assumption that $\theta$ is generic ensures that all of the $\lambda_{i}$ are nonzero. The condition that the $\lambda_{i}$ are nonzero for each column basis of $A$ is equivalent to the condition that every $\theta$-semistable point is stable.
} 
Proof. After reordering the coordinates, we may assume that $x_{\sigma}=\left(x_{1} x_{2} \cdots x_{k}\right) \times$ $\left(y_{k+1} y_{k+2} \cdots y_{d}\right)$ for some $k$ with $0 \leq k \leq d$. Since $A$ has rank $d$, we may also perform row operations over $\mathbb{Z}$ to ensure that $a_{i i} \neq 0$ for $i \leq d$. Then on $U_{\sigma}$ the hypertoric equations $\left\{\sum a_{i j} x_{j} y_{j}\right\}_{i=1}^{d}$ can be rewritten as $x_{i}+\left(1 / y_{i}\right) \sum_{j \neq i}\left(a_{i j} / a_{i i}\right) x_{j} y_{j}$ if $i>k$ and $y_{i}+\left(1 / x_{i}\right) \sum_{j \neq i}\left(a_{i j} / a_{i i}\right) x_{j} y_{j}$ if $i \leq k$. Then we can define an isomorphism $\left(\mu^{-1}(0) \cap U_{\sigma}\right) \times \mathbb{A}^{d} \rightarrow U_{\sigma}$ where $T$ acts trivially on the second factor by the formula

$$
\begin{aligned}
& \left(\left(x_{1}, \ldots, x_{n}, y_{1}, \ldots, y_{n}\right),\left(z_{1}, \ldots, z_{d}\right)\right) \\
& \mapsto\left(x_{1}, \ldots, x_{k}, \frac{z_{k+1}}{y_{k+1}}, \ldots, \frac{z_{d}}{y_{d}},\right. \\
& \left.\quad x_{d+1}, \ldots, x_{n}, \frac{z_{1}}{x_{1}}, \ldots, \frac{z_{k}}{x_{k}}, y_{k+1}, \ldots, y_{n}\right) .
\end{aligned}
$$

Observe that if $Z$ is a $\mathbb{G}_{m}^{2 n}$-invariant irreducible closed subset of $\mathbb{A}^{2 n}$, then $Z$ (with its reduced scheme structure) is defined by linear equations. Thus, if $W \subset U_{\sigma(C, \theta)}$ is a nonempty irreducible $\mathbb{G}_{m}^{2 n}$-invariant closed subset transverse to $\mu^{-1}(0)$, then $W=U_{\sigma} \cap V\left(x_{i_{1}}, \ldots, x_{i_{l}}, y_{j_{1}}, \ldots, y_{j_{m}}\right)$ for some sets indices $\left\{i_{1}, \ldots, i_{l}\right\},\left\{j_{1}, \ldots, j_{m}\right\}$, with $\left\{i_{1}, \ldots, i_{l}\right\} \cap\{1, \ldots, k\}=\emptyset$ and $\left\{j_{1}, \ldots, j_{m}\right\} \cap\{k+$ $1, \ldots, d\}=\emptyset$.

If $\left\{i_{1}, \ldots, i_{l}\right\} \cap\{k+1, \ldots, d\}=\emptyset$ and $\left\{j_{1}, \ldots, j_{m}\right\} \cap\{1, \ldots, d\}=\emptyset$ as well, then isomorphism (5) restricts to a $T$-equivariant isomorphism $W \rightarrow(W \cap$ $\left.\mu^{-1}(0)\right) \times \mathbb{A}^{d}$.

If this is not the case, then we can assume, without loss of generality, that $j_{1} \in\{1, \ldots, d\}$, and after reordering coordinates, we have that $j_{1}=1$. Let $V_{1} \subset V$ be the submodule where $x_{1}=0$, and let $A^{\prime}$ be the matrix obtained by deleting the first column of $A$. Then $W$ is a closed subset of $\mathbb{G}_{m} \times\left(V_{1} \times V_{1}^{*}\right)$, and the hypertoric equations $\sum a_{i j} x_{j} y_{j}$ restrict on $V_{1} \times V_{1}^{*}$ to the equations for $\mathcal{Y}\left(A^{\prime}, \theta\right)$ in $\mathcal{X}\left(A^{\prime \pm}, \theta\right)$. The transversality assumption ensures that the hypertoric equations do not degenerate on $W$. Therefore, by induction on $\operatorname{dim} V$ we can conclude that the conclusion of the lemma holds for the embedding $\mu^{-1}(0) \cap W \hookrightarrow W$.

Proof of Theorem 5.5. The proof is similar to the proof of [HS, Thm. 1.1]. Since $\mathbf{X}\left(A^{ \pm}, \theta\right)$ is a semiprojective toric variety, it is the toric variety of a fan $\Sigma_{\theta}$ in a lattice $N$ that has full dimensional support. A vector $v \in N$ determines a oneparameter subgroup $\lambda_{v}$ that acts on the toric variety $\mathbf{X}\left(A^{ \pm}, \theta\right)$. If $v$ is in the support of the fan $\Sigma_{\theta}$, then the action on $\mathbf{X}\left(A^{ \pm}, \theta\right)$ is filterable. This means that we can order the fixed components for the action of $\lambda_{v}$ so that the Bialynicki-Birula decomposition of $\mathbf{X}\left(A^{ \pm}, \theta\right)$ with respect to this action gives a filtration

$$
\emptyset=\mathbf{U}_{0} \subset \mathbf{U}_{1} \subset \cdots \subset \mathbf{U}_{r}=\mathbf{X}\left(A^{ \pm}, \theta\right)
$$

such that for every $i \geq 1$, the set $\mathbf{U}_{i} \backslash \mathbf{U}_{i-1}$ is a closed subset of $\mathbf{U}_{\sigma}$ where $\sigma$ is a maximal cone in the fan of $X$.

Now if $\lambda_{v}$ is the one-parameter subgroup corresponding to the sum of the generators for the rays in the fan $\Sigma_{\theta}$, then $\lambda_{v}$ is the image of the diagonal oneparameter subgroup of the torus $\mathbb{G}_{m}^{2 n}$ under the quotient map $\mathbb{G}_{m}^{2 n} \rightarrow \mathbb{G}_{m}^{2 n-d}$ [HS, 
Proof of Lemma 6.5]. Since the hypertoric equations are homogeneous in the variables $x_{1}, \ldots, x_{n}, y_{1}, \ldots, y_{n}$, it follows that the quotient $\mathbf{Y}(A, \theta)$ is invariant under the action of $\lambda_{v}$.

As observed by Hausel and Sturmfels, the fixed loci for the $\lambda_{v}$ actions on $\mathbf{X}\left(A^{ \pm}, \theta\right)$ and $\mathbf{Y}(A, \theta)$ are equal since these two semiprojective varieties have a common core.

The Białynicki-Birula decomposition of $\mathbf{Y}(A, \theta)$ gives a filtration

$$
\emptyset=\mathbf{V}_{0} \subset \mathbf{V}_{1} \subset \cdots \subset \mathbf{V}_{r}=\mathbf{Y}(A, \theta)
$$

such that $\mathbf{V}_{i} \backslash \mathbf{V}_{i-1}$ is closed in $\mathbf{U}_{i} \backslash \mathbf{U}_{i-1}$. Moreover, since the hypertoric equations have positive weight with respect to the action of $\lambda_{v}$, the codimension of a component of the Białynicki-Birula decomposition of $\mathbf{Y}(A, \theta)$ in the corresponding component of the Białynicki-Birula decomposition of $\mathbf{X}\left(A^{ \pm}, \theta\right)$ is constant.

By construction of $\mathbf{X}\left(A^{ \pm}, \theta\right)$ as a geometric invariant theory quotient, the inverse image of the affine toric open subset $\mathbf{U}_{\sigma}$ is an open set $U_{\sigma(C, \theta)}$ for some column basis $C$ of $A$. Likewise, any torus-invariant closed subset of $\mathbf{W} \subset \mathbf{U}_{\sigma}$ appearing in the filtration corresponds to a $\mathbb{G}_{m}^{2 n}$ invariant closed subset $W \subset U_{\sigma(C, \theta)}$ that is transverse to $\mu^{-1}(0)$.

By Proposition 5.6 there is a $T$-equivariant isomorphism $\left(\mu^{-1}(0) \cap W\right) \times$ $\mathbb{A}^{d} \rightarrow W$ where $T$ acts trivially on second factor. Hence, the pullbacks $\iota^{*}$ and $i^{*}$ induce isomorphisms of (higher) Chow groups $\mathrm{CH}_{j}\left(\mathcal{U}_{i+1} \backslash \mathcal{U}_{i}, k\right) \rightarrow$ $\mathrm{CH}_{j-d}\left(\mathcal{V}_{i+1} \backslash \mathcal{V}_{i}, k\right)$ and $i^{*}: \mathrm{CH}_{j}\left(\mathbf{U}_{i+1} \backslash \mathbf{U}_{i}, k\right) \rightarrow \mathrm{CH}_{j-d}\left(\mathbf{V}_{i+1} \backslash \mathbf{V}_{i}, k\right)$ for all for all nonnegative integers $j, k$ where $\mathcal{U}_{i}=\left[U_{i} / T\right]$ and $\mathcal{V}_{i}=\left[V_{i} / T\right]$.

Using induction on the stratifications $\mathbf{U}$. and $\mathbf{V}$. and the localization long exact sequence for higher Chow groups, we see that pullback of (higher) Chow groups $i^{*}: \mathrm{CH}_{*}\left(\mathbf{X}\left(A^{ \pm}, \theta\right), k\right) \rightarrow \mathrm{CH}_{*}(\mathbf{Y}(A, \theta), k)$ is an isomorphism.

The same argument also works for the (higher) $K$-theory of coherent sheaves.

REMARK 5.7. Although this result is a Chow group analogue of Hausel and Sturmfels's earlier result on cohomology [HS], the Chow and cohomology groups of a singular toric variety need not be equal. Also observe that the same methods can be used to show that there are corresponding isomorphisms in algebraic $K$-theory.

REMARK 5.8. The proof of Theorem 5.5 makes crucial use of the fact that a Lawrence toric variety is projective over the affine toric variety $\operatorname{Spec}\left(V \times V^{*}\right)^{T}$ since this condition implies that it has a stratification by unions of torus orbits. It would be interesting to give an example where the conclusion of Theorem 5.5 fails for a non-quasi-projective quotient of an open set in $\left(V \times V^{*}\right)$ on which $T$ acts properly; cf. [dMM, Rem. 3.3].

Proof of Corollary 2.9. Again, by Theorem 2.7 it suffices to show that the pullback $(I \iota)^{*}: \mathrm{CH}^{*}\left(I \mathcal{X}\left(A^{ \pm}, \theta\right)\right) \rightarrow \mathrm{CH}^{*}(I \mathcal{Y}(A, \theta))$ is an isomorphism of Abelian groups. Unlike the proof of Corollary 2.8, this not immediate because $\mathcal{X}\left(A^{ \pm}, \theta\right)$ and $\mathcal{Y}(A, \theta)$ are not vector bundles over a common base. 
To prove this isomorphism, we fix some notation. Let $X\left(A^{ \pm}, \theta\right)=(V \times$ $\left.V^{*}\right)^{s}$ and $Y(A, \theta)=\mu^{-1}(0) \cap X\left(A^{ \pm}, \theta\right)$. Then $\mathcal{X}\left(A^{ \pm}, \theta\right)=\left[X\left(A^{ \pm}, \theta\right) / T\right]$ and $\mathcal{Y}\left(A^{ \pm}, \theta\right)=\left[Y\left(A^{ \pm}, \theta\right) / T\right]$.

Observe that $I_{T}\left(X\left(A^{ \pm}, \theta\right)\right)=\bigsqcup_{\{g \in T|| g \mid<\infty\}} X\left(A^{ \pm}, \theta\right)^{g}$ because $T$ is diagonalizable and acts properly. Hence,

$$
I \mathcal{X}\left(A^{ \pm}, \theta\right)=\left[I_{T}\left(X\left(A^{ \pm}, \theta\right)\right) / T\right]=\coprod_{\{g \in T|| g \mid<\infty\}}\left[X\left(A^{ \pm}, \theta\right)^{g} / T\right],
$$

and a similar statement holds for $I \mathcal{Y}(A, \theta)$. (Note that all disjoint sums are finite since if $T$ acts properly on a space $X$, then $X^{g}=\emptyset$ for all but finitely many $g \in T$.)

Observe that if $V$ is a $T$-module and $g \in T$, then $\left(V^{*}\right)^{g}=\left(V^{g}\right)^{*}$ so $(V \times$ $\left.V^{*}\right)^{g}=V^{g} \times\left(V^{g}\right)^{*}$ as $T$-modules. Also, if $\theta$ is a character, then

$$
\left(V^{g} \times\left(V^{g}\right)^{*}\right)^{s}=\left(V^{g} \times\left(V^{g}\right)^{*}\right) \cap\left(V \times V^{*}\right)^{s},
$$

where stability is taken with respect to the character $\theta$. Since the action of $T$ on $V$ is diagonalized, the submodule $V^{g}$ is obtained by setting coordinates $x_{i_{1}}, \ldots, x_{i_{k}}$ to zero. Hence, $X\left(A^{ \pm}, \theta\right)^{g}=X\left(A_{g}^{ \pm}, \theta\right)$ where $A_{g}$ is the matrix obtained from $A$ by deleting the $i_{1}, \ldots, i_{k}$ th columns. (Note that $k$ and the integers $i_{1}, \ldots, i_{k}$ depend on $g$.) Hence, $I \mathcal{X}\left(A^{ \pm}, \theta\right)=\bigsqcup_{\{g \in T|| g \mid<\infty\}} \mathcal{X}\left(A_{g}^{ \pm}, \theta\right)$. A similar argument shows that $I \mathcal{Y}=\bigsqcup_{\{g \in T|| g \mid<\infty\}} \mathcal{Y}\left(A_{g}, \theta\right)$. Hence, by Theorem 5.5 the pullback $I \iota^{*}: \mathrm{CH}^{*}\left(I \mathcal{X}\left(A^{ \pm}, \theta\right)\right) \rightarrow \mathrm{CH}^{*}(I \mathcal{Y}(A, \theta))$ is an isomorphism.

We conclude with a conjecture.

CONJECTURE 5.9. If $\tilde{X}$ is a canonical (hence toric) resolution of singularities of the Lawrence toric variety $X(A, \theta)$ and $\tilde{Y}=Y(A, \theta) \times_{X(A, \theta)} \tilde{X}$, then the pullback $\mathrm{CH}^{*}(\tilde{X}) \rightarrow \mathrm{CH}^{*}(\tilde{Y})$ is an isomorphism.

Acknowledgments. The author is grateful to Daniel Bergh and Daniel Lowengrub for helpful discussions. The author is also grateful to referee for a number of helpful comments.

\section{References}

[AOV] D. Abramovich, M. Olsson, and A. Vistoli, Tame stacks in positive characteristic, Ann. Inst. Fourier (Grenoble) 58 (2008), no. 4, 1057-1091.

[Art] M. Artin, Algebraic approximation of structures over complete local rings, Publ. Math. Inst. Hautes Études Sci. 36 (1969), 23-58.

[BD] R. Bielawski and A. S. Dancer, The geometry and topology of toric hyperkähler manifolds, Comm. Anal. Geom. 8 (2000), no. 4, 727-760.

[Blo] S. Bloch, Algebraic cycles and higher K-theory, Adv. Math. 61 (1986), no. 3, 267-304.

[Buo] F. Buonerba, Functorial resolution of tame quotient singularities in positive characteristic, arXiv:1511.00550.

[CR] W. Chen and Y. Ruan, Orbifold Gromov-Witten theory, Orbifolds in mathematics and physics, Contemp. Math., 310, pp. 25-85, Amer. Math. Soc., Providence, RI, 2002. 
[dMM] M. A. de Cataldo, L. Migliorini, and M. Mustata, The combinatorics and topology of proper toric maps, arXiv: 1407.3497.

[EG1] D. Edidin and W. Graham, Equivariant intersection theory, Invent. Math. 131 (1998), no. 3, 595-634.

[EG2] Algebraic cycles and completions of equivariant $K$-theory, Duke Math. J. 144 (2008), no. 3, 489-524.

[EJK1] D. Edidin, T. J. Jarvis, and T. Kimura, Logarithmic trace and orbifold products, Duke Math. J. 153 (2010), no. 3, 427-473.

[EJK2] A plethora of inertial products, Ann. K-Theory 1 (2016), no. 1, 85-108.

[Ful] W. Fulton, Intersection theory, Springer-Verlag, Berlin, 1984.

[EGA4] A. Grothendieck and J. Dieudonné, Élements de Géométrie Algébrique IV. Étude locale des schemas et des morphismes de schémas, Publ. Math. Inst. Hautes Études Sci. 20, 24, 28, 32 (1964, 1965, 1966, 1967).

[HS] T. Hausel and B. Sturmfels, Toric hyperKähler varieties, Doc. Math. 7 (2002), 495-534 (electronic).

[JKK] T. J. Jarvis, R. Kaufmann, and T. Kimura, Stringy K-theory and the Chern character, Invent. Math. 168 (2007), no. 1, 23-81.

[JT1] Y. Jiang and H.-H. Tseng, Note on orbifold Chow ring of semi-projective toric Deligne-Mumford stacks, Comm. Anal. Geom. 16 (2008), no. 1, 231-250.

[JT2] The orbifold Chow ring of hypertoric Deligne-Mumford stacks, J. Reine Angew. Math. 619 (2008), 175-202.

[KM] S. Keel and S. Mori, Quotients by groupoids, Ann. of Math. (2) 145 (1997), no. 1, 193-213.

[Kol] J. Kollár, Lectures on resolution of singularities, Ann. of Math. Stud., 166, Princeton University Press, Princeton, NJ, 2007.

[Kre] A. Kresch, Cycle groups for Artin stacks, Invent. Math. 138 (1999), no. 3, 495536.

[Lev] M. Levine, Techniques of localization in the theory of algebraic cycles, J. Algebraic Geom. 10 (2001), no. 2, 299-363.

[Low] D. Lowengrub, A cancellation theorem for Segre classes, arXiv:1503.01569.

[Pro1] N. Proudfoot, All the GIT quotients at once, Trans. Amer. Math. Soc. 363 (2011), no. 4, 1687-1698.

[Pro2] N. J. Proudfoot, A survey of hypertoric geometry and topology, Toric topology, Contemp. Math., 460, pp. 323-338, Amer. Math. Soc., Providence, RI, 2008.

[Sum] H. Sumihiro, Equivariant completion, J. Math. Kyoto Univ. 14 (1974), 1-28.

[Tho] R. W. Thomason, Comparison of equivariant algebraic and topological $K$ theory, Duke Math. J. 53 (1986), no. 3, 795-825.

Department of Mathematics

University of Missouri-Columbia

Columbia, MO 65211

USA

edidind@missouri.edu 